\title{
3D LES simulations of a static and vertically free-to-oscillate 4:1 rectangular cylinder: effects of the grid resolution
}

\author{
A. J. Álvarez ${ }^{\mathrm{a}}$, F. Nieto ${ }^{\mathrm{a}}$, D. T. Nguyen ${ }^{\mathrm{b}}$, J. S. Owen ${ }^{\mathrm{b}}$ \& S. Hernández ${ }^{\mathrm{a}}$. \\ a) School of Civil Engineering, University of La Coruña, Campus de Elviña, La Coruña, 15071, Spain. \\ b) School of Civil Engineering, University of Nottingham, Faculty of Engineering, Department of Civil \\ Engineering, University Park, Nottingham NG7 2RD, UK.
}

\begin{abstract}
Vortex induced vibration (VIV) is an important phenomenon which appears in flexible structures immersed in a moving fluid. This oscillation is self-sustained and self-limited, but VIV might cause fatigue damage and affect the structure's serviceability.

In the present study, the aerodynamics of the flow fields around a static and vertically free-to-oscillate 4:1 rectangular cylinder are analysed by means of 3D LES simulations, adopting the OneEqEddy viscosity model. Integral parameters, pressure distributions, amplitudes of oscillation, coherences and correlations are obtained and compared with the available experimental data. Aiming to ascertain the impact of the boundary conditions and the grid resolution on the accuracy of results, five cases adopting 3 different meshes including two different spanwise discretisations have been considered. When studying the aerodynamics of the cylinder in static conditions, the influence of the spatial discretisation is very limited, and the agreement with experimental data is fairly good. On the other hand, for the free-to-oscillate cylinder, the structural response is dramatically dependent on the spanwise discretisation. The maximum amplitude of the structural response decreases as the mesh resolution increases, providing a closer fit with the experimental data. Also, the spanwise correlation of pressures is studied, finding remarkable differences depending on the level of spatial discretisation.
\end{abstract}

Keywords: 3D LES, rectangular cylinder, vortex shedding, vortex induced vibrations, spanwise discretisation, correlations, coherence, force coefficients, Strouhal number, pressure coefficient distribution.

\section{Introduction}

Rectangular cylinders are a common geometry used in many structural members. Moreover, certain width to depth ratios (4:1 or 5:1 for instance) can be used in fundamental studies to represent a generic bridge deck. These geometries present fixed flow separation points and may show large amplitudes of oscillation at lock-in (Marra et al., 2011).

Experimental campaigns of rectangular cylinders with different width to depth ratios have been carried out for many years for both static and free-to-oscillate configurations. Nakaguchi et al. (1968) studied the variability of the drag coefficient, Strouhal number and pressure coefficient distributions with respect to the width to depth ratios of a static cylinder, ranging from 0.1 to 4.0. Also for static models, Mizota and Okajima (1981) studied the unsteady flow around rectangular cylinders having ratios of 0.4, 1.0, 2.0 and 4.0. Okajima (1983) considered cylinders with ratios ranging from 0.8 to 6 , including in his studies velocity and Strouhal number measurements. Washizu et al. (1978) studied the aeroelastic instabilities of rectangular cylinders of ratios ranging from 1 to 4 in heaving oscillation. Komatsu and Kobayashi (1980) studied the VIV for different geometries, including rectangular cylinders of ratio 2, 3, 4 and 5. In recent years Marra et al. (2015) studied the influence of damping on the VIV structural response of a 4:1 rectangular cylinder and proposed an empirical expression formulating this relationship.

These types of geometries have also been studied numerically. Ohya et al. (1992) studied the vortex shedding over rectangular cylinders with ratios ranging from 3 to 9 . In the recent years, thanks to the BARC initiative, the ratio 5:1 rectangular cylinder has been extensively studied by means of both numerical and experimental 
research (Bruno et al., 2014; Ricci et al., 2017). Shimada and Ishihara (2012) studied the VIV for rectangular cylinders of ratio 2 and 4 by means of numerical and experimental techniques, meanwhile Nguyen et al. (2018) studied a 5:1 rectangular cylinder. In LES simulations researchers have used a wide range of spanwise discretisations. For instance, for the case of the 5:1 rectangular cylinder, in Bruno et al. (2014) a compendium of the discretisations used by different researches is presented, ranging from $\delta z / B=0.0025$ to 0.042 . In a more recent paper (Daniels et al., 2016), a spanwise discretisation of $\delta z / B=0.025$ for a 4:1 rectangular cylinder undergoing free heave or torsional vibrations was used. This is an important parameter as in many cases these meshes are built by extrusion along the spanwise direction, hence impacting the overall number of elements. To the best knowledge of the authors, no sensitivity study of this key parameter in the VIV response of rectangular cylinders has been reported.

LES CFD simulations are a powerful tool to study the 3D flow field around a body submerged in a fluid. In computational wind engineering practice, the effect of the spatial resolution in the accuracy of the simulations must be carefully assessed. The level of refinement of the grid along a flow domain section orthogonal to the axis of the body (plane $X Y$ in figure 1) and, very remarkably, over the spanwise direction, plays a key role in the ability of the computational simulations to match experimental tests. In that sense, the present work attempts to increase the knowledge about the different phenomena taking place over a rectangular cylinder of ratio 4:1, and the impact of different mesh discretisations, for the static and vertically free to oscillate cases. Grid resolution studies are scarce in the literature, focusing mainly on fixed bodies (Bruno et al., 2012), hence to the author's knowledge there are no available studies dealing with fluid structure interaction simulations using 3D LES. In this piece of research, it has been found that vortex induced vibration (VIV), is a particularly demanding problem in terms of spatial discretisation, because of the need to properly simulate the flow correlation over the cylinder's span, which deeply impacts the structural response of the system. Previous studies dealing with numerical simulations of a 4:1 rectangular cylinder by means of LES simulations can be found in Yu and Kareem (1998), Sohankar (2008), Sarwar and Ishihara (2010) or Daniels et al. (2016).

\section{Formulation}

\subsection{Governing equations}

The unsteady flow field around the cylinder is modelled by the Navier-Stokes equations, which in the LES approach are spatially filtered by the cell size, as follows (Bruno et al., 2010):

$$
\begin{aligned}
& \frac{\partial \overline{u_{i}}}{\partial x_{i}}=0, \\
& \frac{\partial \overline{u_{i}}}{\partial t}+\frac{\partial \overline{u_{i} u_{j}}}{\partial x_{j}}=-\frac{1}{\rho} \frac{\partial \bar{p}}{\partial x_{i}}+\frac{\partial}{\partial x_{j}}\left[\nu\left(\frac{\partial \overline{u_{i}}}{\partial x_{j}}+\frac{\partial \overline{u_{j}}}{\partial x_{i}}\right)+\tau_{i j}^{s}\right],
\end{aligned}
$$

where $\bar{u}$ is the filtered velocity, $\bar{p}$ is the filtered pressure, $x$ is the space coordinate, $t$ is the time, $\nu$ is the kinematic viscosity and $\rho$ is the fluid density. Using the Boussinesq assumption, the sub-grid stress tensor is expressed as shown in equation (3). Thus, the system of equations can be closed by adding a transport equation for the kinematic energy $k_{t}$ of the unresolved stresses (Yoshizawa, 1986):

$$
\begin{aligned}
& \tau_{i j}^{S}=\nu_{t}\left(\frac{\partial \overline{u_{i}}}{\partial x_{j}}+\frac{\partial \overline{u_{j}}}{\partial x_{i}}\right), \\
& \frac{\partial k_{t}}{\partial t}+\frac{\partial}{\partial x_{j}}\left(\overline{u_{j}} k_{t}\right)=\frac{\partial}{\partial x_{j}}\left[\left(\nu+\nu_{t}\right) \frac{\partial k_{t}}{\partial x_{j}}\right]+P_{k}-C_{\varepsilon} \frac{k_{t}^{\frac{3}{2}}}{l_{\varepsilon}},
\end{aligned}
$$

where $P_{k}=2 \nu_{t} \overline{S_{i j} S_{i j}}, \nu_{t}=C_{k} l_{k} k_{t}^{\frac{1}{2}}$, with $C_{\varepsilon}=1.05, C_{k}=0.07$ and $l_{\varepsilon}=l_{k}=\Delta . \Delta$ is the characteristic spatial length of the filter, related to the mesh size and defined as the cubic root of the mesh cell volume. For 
the modelling of the flow in the turbulent boundary layer a damped filter width $\delta$ according to the Van Driest approach is introduced:

$$
\delta=\min \left\{\Delta, \frac{k}{C_{\Delta}} y\left(1-\exp \left(-\frac{y^{+}}{A^{+}}\right)\right)\right\},
$$

where $k=0.4187$ is the Von Karman constant, $C_{\Delta}=0.158, A^{+}=26$ is the Van Driest constant, $y$ the distance to the wall, $y^{+}=\left(u_{\tau} y\right) / \nu$ the non dimensional wall unit and $u_{\tau}$ the shear velocity (de Villiers, 2006). In other words, for the boundary layer the characteristic length of the filter is adapted locally in space and time.

For the present study the LES model OneEqEddy provided by the open-source CFD software OpenFOAM was selected.

\subsection{Modelling and computational approach}

The filtered Navier-Stokes equations are solved by means of the CFD software OpenFOAM. The diffusive terms were discretised by using the second order central difference scheme while the first/second order upwind differencing scheme was applied for the convective terms. Advancement in time was performed by the second order backward scheme. Finally, the pressure-velocity coupling was solved by the PIMPLE algorithm. The maximum Courant $(\mathrm{Co})$ number was limited to 1 at every time step. In the dynamic simulations, the Arbitrary Lagrangian Eulerian algorithm was applied to incorporate the movement of the mesh into the governing fluid equations. The cylinder was restrained allowing displacements only in the heave degree of freedom, and its oscillation was modelled by a single-degree-of-freedom mass-spring-damper system. The coupling between the fluid, structure and dynamic mesh was achieved by the use of the conventional serial staggered scheme.

The overall computational domain size is shown in figure 1a, and its dimensions are presented in table 1, which are very similar to the ones reported in Bruno et al. (2014) for various 5:1 rectangular cylinder simulations. In order to better control the elements growth and the mesh refinement in the regions of interest, the fluid domain in the $X Y$ plane was subdivided in four zones, as described in figure 1b. Zone $\mathbf{K}$ is the so called buffer zone, which is the zone surrounding the rectangular cylinder, where very important phenomena take place: flow separation and reattachment, as well as vortex shedding. Zone $\mathbf{L}$ is the wake zone, a narrow band downwind the rectangular cylinder in which the vortices shed from the cylinder evolve and eventually dissipate. Finally, Zones $\mathbf{M}$ and $\mathbf{N}$ define the overall dimension of the fluid domain, which is large enough to minimise the influence of the boundary conditions on the flow around the cylinder. Also, Zones $\mathbf{M}$ and $\mathbf{N}$ control the size of the elements far away from the rectangular cylinder.

At the inlet, Dirichlet conditions were imposed for the velocity and turbulent kinetic energy, meanwhile Neumann conditions were imposed for the pressure. At the outlet, Dirichlet conditions were applied to the pressure, and Neumann conditions to the velocity and turbulent kinetic energy. For the upper, lower and lateral faces, two different boundary conditions were applied in different cases: periodic and symmetric. All the simulations were conducted imposing smooth flow condition $(I=0 \%, I$ is the turbulence intensity) at the inlet. For the rectangular cylinder walls, no-slip and no-penetration boundary conditions were applied (Wilcox, 2012).

The characteristics of the different meshes considered in this work are presented in the table 2. Cases C01 and $\mathrm{C} 02$ use the same mesh but they differ in the boundary conditions imposed on the lateral, top and bottom sides. Meanwhile, the difference between cases C02 and C03 is that case C03 has a finer discretisation on the $X Y$ plane. Case $\mathrm{C} 04$ has the double number of elements in the spanwise direction compared to case C03, while keeping the same discretisation in the $X Y$ plane. Finally, the difference between the cases C04 and C05, is that the latter one has a finer discretisation on the $X Y$ plane (see tables 2 and 3 ). Images from the mesh of the case C05 are shown in figures $2 \mathrm{a}$ to $2 \mathrm{~d}$. 


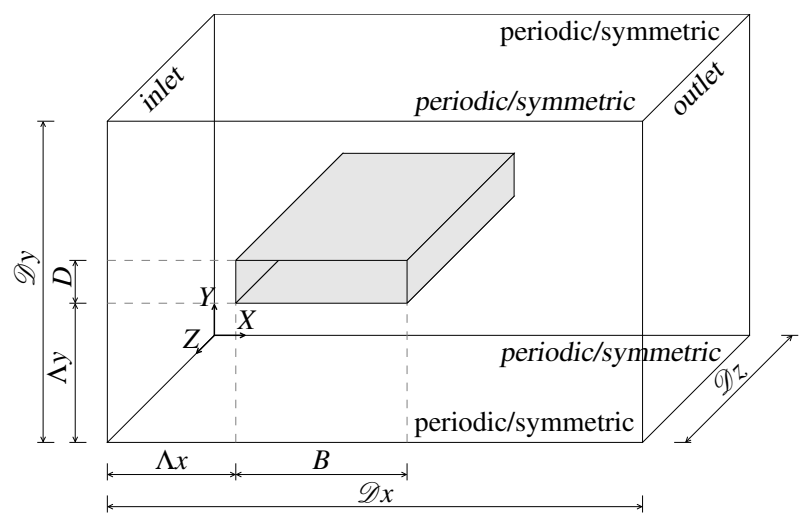

(a)

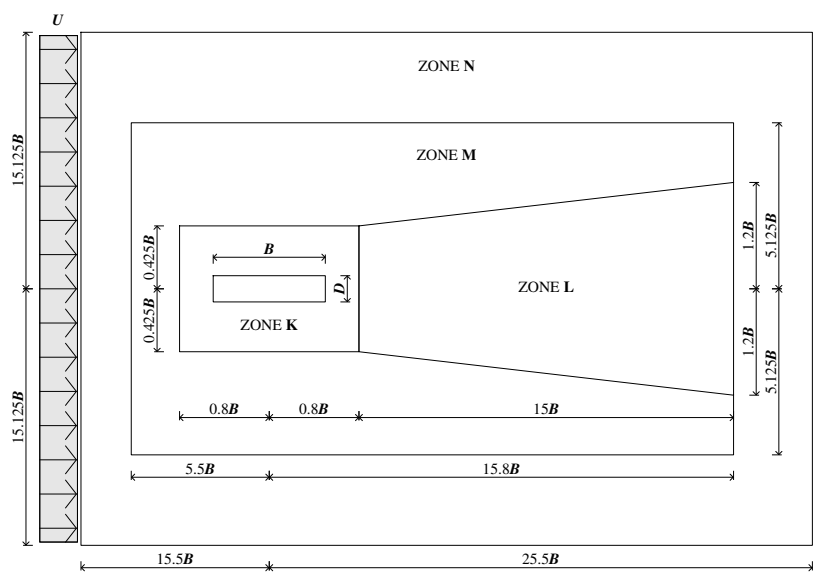

(b)

Figure 1: (a) Computational fluid domain and (b) mesh zones subdivisions in the $X Y$ plane.

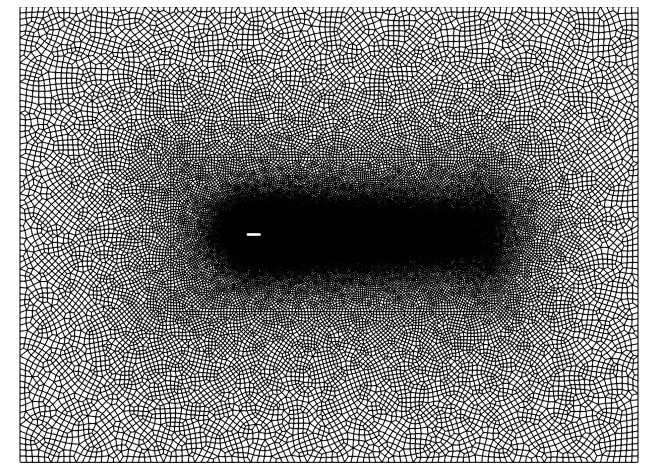

(a) Overall domain.

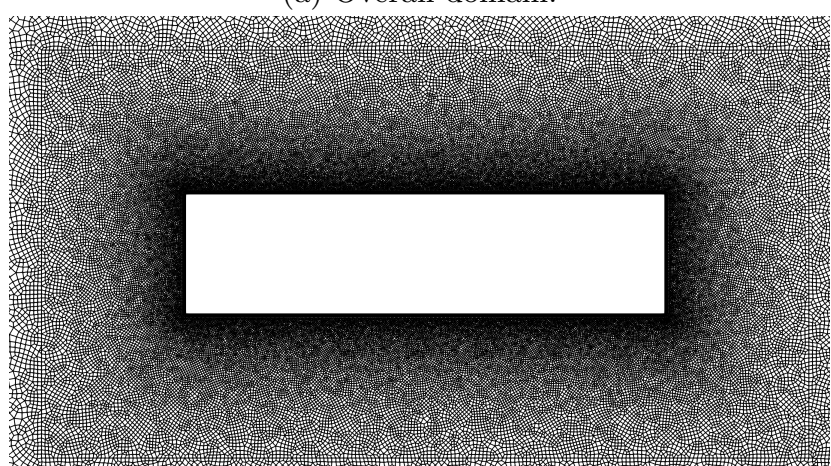

(c) Zone $\mathbf{K}$.

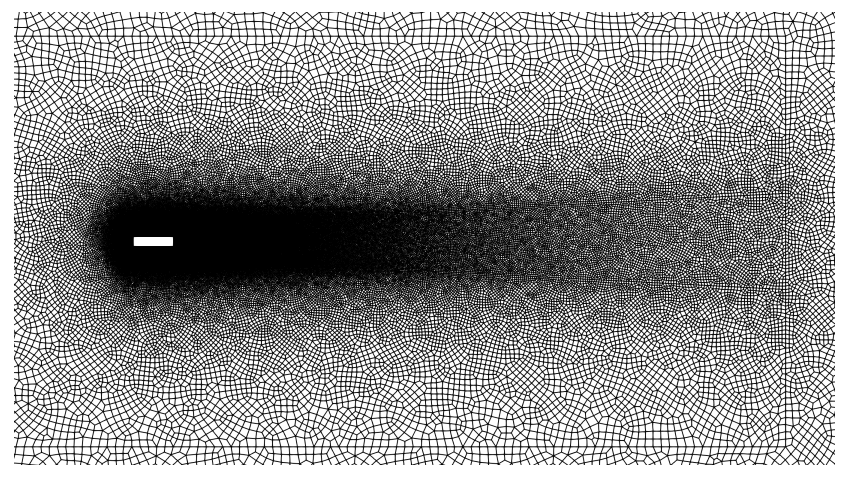

(b) Zones $\mathbf{K}, \mathbf{L}$ and $\mathbf{M}$.

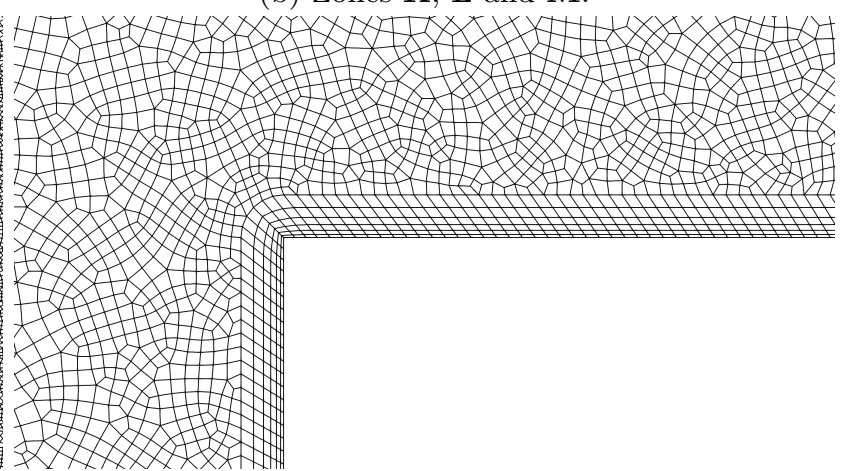

(d) Boundary layer mesh.

Figure 2: Computational domain and mesh details for the case C05. 
Table 1: Domain Dimensions. $B$ is the cylinder width and $D$ is the cylinder height.

\begin{tabular}{cccccc}
\hline$D$ & $\Lambda x$ & $\Lambda y$ & $\mathscr{D} x$ & $\mathscr{D} y$ & $\mathscr{D} z$ \\
\hline$B / 4$ & $15 B$ & $15 B$ & $41 B$ & $30.25 B$ & $B$ \\
\hline
\end{tabular}

Table 2: Mesh properties.The parameter $y_{1}$ is the height of the first element of the boundary layer (BL), $B$ is the width of the rectangular cylinder, $x_{1}$ is the length of first element in the BL, $r$ is the growth ratio of the elements in the BL, $n_{B L}$ is the number of layers forming the BL and $y_{B L}$ is the total height of the BL, $\delta_{z}$ is the cell size in the spanwise dimension, $n_{z}$ is the number of elements in the spanwise dimension and $\mathrm{BC}$ are the type of boundary conditions applied to the lateral, upper and lower faces.

\begin{tabular}{cccccccccccc}
\hline Case & $y_{1} / B$ & $x_{1} / y_{1}$ & $r$ & $n_{B L}$ & $y_{B L} / B$ & $\delta_{z} / B$ & $n_{z}$ & $X Y$ cells & Total cells & BL cells & BC \\
\hline C01 & $6.67 \cdot 10^{-4}$ & 4 & 1.32 & 6 & $8.94 \cdot 10^{-3}$ & $4.17 \cdot 10^{-2}$ & 24 & 42656 & 1023744 & 134784 & periodic \\
C02 & $6.67 \cdot 10^{-4}$ & 4 & 1.32 & 6 & $8.94 \cdot 10^{-3}$ & $4.17 \cdot 10^{-2}$ & 24 & 42656 & 1023744 & 134784 & symmetric \\
C03 & $6.67 \cdot 10^{-4}$ & 4 & 1.32 & 6 & $8.94 \cdot 10^{-3}$ & $4.17 \cdot 10^{-2}$ & 24 & 95462 & 2291088 & 134784 & symmetric \\
C04 & $6.67 \cdot 10^{-4}$ & 4 & 1.32 & 6 & $8.94 \cdot 10^{-3}$ & $2.08 \cdot 10^{-2}$ & 48 & 95462 & 4582176 & 269568 & symmetric \\
C05 & $6.67 \cdot 10^{-4}$ & 4 & 1.32 & 6 & $8.94 \cdot 10^{-3}$ & $2.08 \cdot 10^{-2}$ & 48 & 146953 & 7053744 & 269568 & symmetric \\
\hline
\end{tabular}

Table 3: Number of elements of the different mesh zones for all the meshes studied on the $X Y$ plane.

\begin{tabular}{ccccc}
\hline Case & Zone $\mathbf{K}$ & Zone $\mathbf{L}$ & Zone $\mathbf{M}$ & Zone N \\
\hline C01 & 15739 & 6102 & 11049 & 4150 \\
C02 & 15739 & 6102 & 11049 & 4150 \\
C03 & 32226 & 18239 & 29896 & 9485 \\
C04 & 32226 & 18239 & 29896 & 9485 \\
C05 & 52710 & 31248 & 42599 & 14780 \\
\hline
\end{tabular}

In table 4, the flow domain and discretisation characteristics of several numerical simulations, available in the literature, for ratio 4 and 5 rectangular cylinders are presented. It may be appreciated that the dimensions of the flow domain and the level of discretisation used in this work are in the range of state-of-the-art simulations conducted by other researchers.

Table 4: Characteristics of LES simulations of rectangular cylinders of ratio 4 and 5 available in the literature.

\begin{tabular}{cccccccccccccc}
\hline & $B / D$ & $\operatorname{Re}_{D}$ & $I$ & $\Lambda x / B$ & $\Lambda y / B$ & $\mathscr{D} x / B$ & $\mathscr{D} y / B$ & $\mathscr{D} z / B$ & $y_{1} / B$ & $\delta_{x} / B$ \\
\hline Sohankar (2008) & 4 & $1.0 \cdot 10^{5}$ & 0 & 1.875 & - & 6.875 & - & 1 & - & var. & 0.04 & periodic \\
Daniels et al. (2016) & 4 & $0.4 \cdot 10^{4}$ & $0-12$ & - & 2.4 & 16.65 & 5 & 3.325 & $1.2 \cdot 10^{-3}$ & - & 0.025 & periodic \\
Arslan et al. (2011) & 5 & $0.26 \cdot 10^{5}$ & 0 & 10 & 10 & 23 & 20.2 & 1 & $2.0 \cdot 10^{-4}$ & $4.0 \cdot 10^{-3}$ & 0.04 & periodic \\
Bruno et al. (2010,2011) & 5 & $0.4 \cdot 10^{5}$ & 0 & 15 & 15 & 41 & 30.2 & $1,2,4$ & $5.0 \cdot 10^{-4}$ & $2.0 \cdot 10^{-3}$ & $0.042-0.01$ & periodic \\
Grozescu et al. (2011b) & 5 & $0.4 \cdot 10^{5}$ & 0 & 15 & 15 & 41 & 30.2 & 1 & $5.0-2.5 \cdot 10^{-4}$ & $10^{-2}-2.0 \cdot 10^{-3}$ & $0.042-0.01$ & periodic \\
Patruno (2016) & 5 & $0.27 \cdot 10^{5}$ & 0 & 16 & 14.9 & 40 & 30 & 2 & $5.0 \cdot 10^{-4}$ & $2.5 \cdot 10^{-3}$ & 0.02 & periodic \\
Ricci et al. (2017) & 5 & $0.55 \cdot 10^{5}$ & $2.9-13.6$ & 16 & 14.9 & 40 & 30 & 2 & $5.0 \cdot 10^{-4}$ & $2.5 \cdot 10^{-3}$ & 0.02 & periodic \\
Nguyen et al. (2018) & 5 & $0.13 \cdot 10^{5}$ & - & 2.5 & 2 & 11 & 4.2 & 3 & $2.0 \cdot 10^{-3}$ & $4.0 \cdot 10^{-3}$ & 0.1 & periodic \\
Present study & 4 & $0.59 \cdot 10^{5}$ & 0 & 15 & 15 & 41 & 30.25 & 1 & $6.67 \cdot 10^{-4}$ & $2.66 \cdot 10^{-3}$ & $0.042-0.021$ & symmetric \\
\hline
\end{tabular}

The values of the $y^{+}$were calculated as in (Bruno et al., 2010), obtaining a mean value $\overline{y^{+}} \simeq 0.65$, and less than a $4 \%$ of the elements around the body have a $y^{+}$value higher than 4 . Further details about the $y^{+}$values of the different cases is provided in table 5 . The maximum $y^{+}$values are mainly located in the vicinity of the corners of the rectangular cylinder. 
Table 5: $y^{+}$values for the different cases.

\begin{tabular}{cccc}
\hline Case & $y_{\text {mean }}^{+}$ & $\% y^{+}>2$ & $\% y^{+}>4$ \\
\hline C01 & 0.6519 & 3.6325 & 1.2821 \\
C02 & 0.6297 & 3.7749 & 1.1129 \\
C03 & 0.6281 & 3.6369 & 0.6455 \\
C04 & 0.6364 & 3.6636 & 0.6833 \\
C05 & 0.6593 & 3.9441 & 1.2731 \\
\hline
\end{tabular}

\subsection{Force Coefficients, Strouhal Number and Pressure Coefficient}

The force coefficients (drag $\left(C_{d}\right)$, lift $\left(C_{l}\right)$ and moment $\left.\left(C_{m}\right)\right)$ along with the Strouhal $(S t)$ number are the so called integral parameters. They are calculated as indicated in equation (6),

$$
C_{d}=\frac{F_{D}}{\frac{1}{2} \rho U^{2} D}, \quad C_{l}=\frac{F_{L}}{\frac{1}{2} \rho U^{2} D}, \quad C_{m}=\frac{M}{\frac{1}{2} \rho U^{2} D^{2}}, \quad S t=\frac{f D}{U} .
$$

where $D$ stands for the cylinder height, $\rho$ is the air density, $U$ is the free-stream velocity, $f$ is the dominant frequency of the lift coefficient, and $F_{D}, F_{L}$ and $M$ are the drag and lift forces and moment per unit of length, which were calculated as the time and spanwise averaging of the integration of the pressure and viscous forces along the rectangular cylinder surface. The sign convention for the movements and force coefficients is presented in figure 3. The positive directions for the lift and moment are the upward direction and clockwise rotation respectively. The positive drag is the along wind direction, which is coincident with the positive $X$-axis in figure 1a.

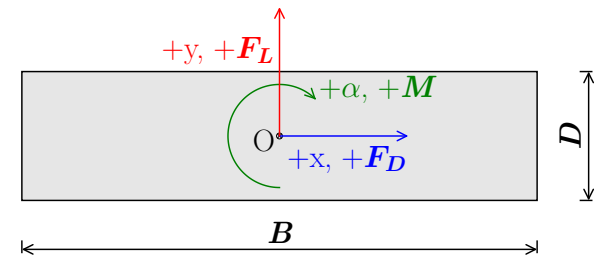

Figure 3: Sign convention.

The standard deviation of the force coefficients is calculated as indicated in equation (7):

$$
\widetilde{C_{i}}=\sqrt{\frac{1}{n} \sum_{k=1}^{n}\left(C_{i k}-\overline{C_{i}}\right)^{2} .}
$$

The mean pressure coefficient $\left(\overline{C_{p}}\right)$ and its standard deviation $\left(\widetilde{C_{p}}\right)$ are calculated as shown by equations $(8)$ and (9).

$$
\begin{aligned}
& \overline{C_{p}}=\frac{\bar{p}}{\frac{1}{2} \rho U^{2}}, \\
& \widetilde{C_{p}}=\frac{\sigma_{p}}{\frac{1}{2} \rho U^{2}} .
\end{aligned}
$$

In the latter equations, $\bar{p}$ is the time averaged pressure and $\sigma_{p}$ is the standard deviation of the pressure. The distributions of $\overline{C_{p}}$ and $\widetilde{C_{p}}$ help to understand the resultant mean force coefficients and the fundamental flow features over the cylinder. 


\section{Results for the static case}

\subsection{Integral parameters and force coefficients correlation.}

The values of the force coefficients and $S t$ number obtained from the simulations where different boundary conditions and mesh discretisations were considered are presented next. For all the simulations, the convergence criteria for the mean drag coefficient and the standard deviation of the drag, lift and moment coefficients, as well as the Strouhal number were based on the values of the residual of the corresponding variables. The residuals were calculated as $\varphi_{\text {res }}=\left|\left(x_{n}-x_{n-1}\right) / x_{n}\right| \cdot 100$ and the simulations were extended until the residual value was less than 5\%, as indicated in (Bruno et al., 2010) ( $n$ is the number of sampling windows). These residuals were calculated for increasing extends of the sampling window $T_{n}$, with $T_{0}=50$ and $T_{n}=T_{n-1}+50$ (in non-dimensional time units $((t U) / D)$ ). This criterion is not appropriate to assess close-to-zero variables, as very small variations imply large residuals. Therefore, for the mean value of the lift and moment coefficients the simulations were extended until the difference between two consecutive sampling windows is less than 0.05 in absolute value (see figure 4).

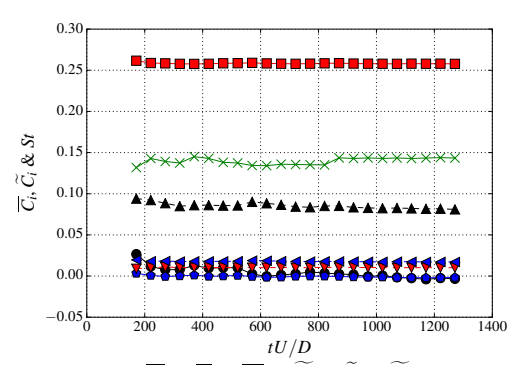

(a)

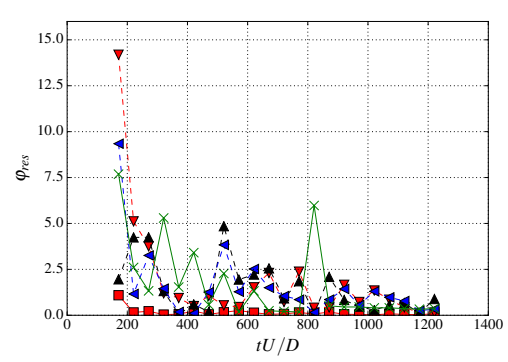

(b)

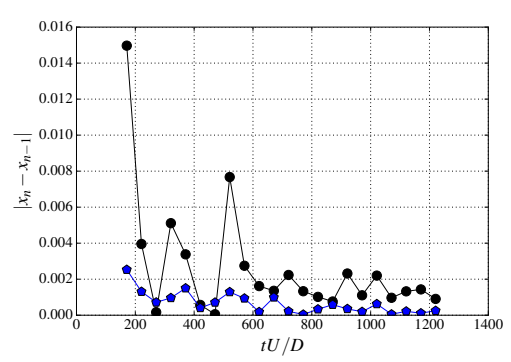

(c)

Figure 4: Force coefficients and Strouhal number (a) evolution, (b) residuals and (c) absolute difference for the case C05.

In table 6 the results obtained for the mean values and standard deviation of the force coefficients and Strouhal number are in good agreement with the experimental and numerical data taken from the literature. It can be noticed a decrease in the standard deviation of the force coefficients with increasing grid resolution in the $X Y$ plane, which is in agreement with results obtained by Grozescu et al. (2011a,b).

Table 6: Force coefficients for the static cases. All data calculated with respect to $B$ except for Re and St calculated with respect to $D$.

\begin{tabular}{ccccccccccccccc}
\hline Case & Method & Re & $I[\%]$ & $\overline{C_{d}}$ & $\overline{C_{l}}$ & $\overline{C_{m}}$ & $\widetilde{C_{d}}$ & $\widetilde{C_{l}}$ & $\widetilde{C_{m}}$ & $S t$ \\
\hline C01 & LES & $0.59 \cdot 10^{5}$ & 0 & 1.1404 & -0.0604 & 0.0288 & 0.0972 & 0.9016 & 0.7312 & 0.135 \\
C02 & LES & $0.59 \cdot 10^{5}$ & 0 & 1.1408 & -0.0592 & 0.0160 & 0.0880 & 0.8172 & 0.6576 & 0.135 \\
C03 & LES & $0.59 \cdot 10^{5}$ & 0 & 1.0708 & 0.0440 & 0.0144 & 0.0684 & 0.5192 & 0.4288 & 0.131 \\
C04 & LES & $0.59 \cdot 10^{5}$ & 0 & 1.0424 & 0.0124 & -0.0080 & 0.0484 & 0.4144 & 0.3392 & 0.138 \\
C05 & LES & $0.59 \cdot 10^{5}$ & 0 & 1.0308 & -0.0264 & 0.0320 & 0.0400 & 0.3128 & 0.2672 & 0.142 \\
Nakaguchi et al. (1968) & Exp. & $0.2-0.6 \cdot 10^{5}$ & - & 1.1192 & - & - & - & - & - & 0.129 \\
Mizota and Okajima (1981) & Exp. & $0.43 \cdot 10^{5}$ & - & - & 0.0700 & - & - & - & - & 0.118 \\
Igarashi (1985) & Exp. & $0.26-0.58 \cdot 10^{5}$ & - & 1.2132 & - & - & - & - & - & 0.135 \\
Vairo (2003) by CSTB & Exp. & $0.5 \cdot 10^{4}$ & - & 1.4240 & -0.2960 & -0.4160 & 0.3240 & 1.1560 & 0.8640 & 0.159 \\
Marra et al. (2011) & Exp. & $0.15-0.57 \cdot 10^{5}$ & - & - & - & - & - & - & - & 0.136 \\
Sarkic (2014) & Exp. & $0.2 \cdot 10^{5}-0.33 \cdot 10^{5}$ & $3-4$ & 0.9240 & 0.2680 & -0.0160 & 0.1400 & 0.5440 & - & 0.129 \\
Yu and Kareem (1998) & LES & $1.0 \cdot 10^{5}$ & - & 1.300 & - & - & 0.1400 & 1.0300 & - & 0.156 \\
Sohankar (2008) & LES & $1.0 \cdot 10^{5}$ & 0 & 1.2024 & - & - & 0.0536 & 0.4636 & - & 0.142 \\
\hline
\end{tabular}


The spanwise correlation of the drag, lift and moment coefficients are presented in figure 5 . The correlation is calculated as shown in equation (10), where $\Delta z$ is the spanwise distance between the two points where the correlation is going to be calculated, and $S$ represents the time-history of the variable of interest.

$$
R_{C_{i}}(\Delta z / D)=\frac{\operatorname{cov}\left(S_{z / D}, S_{(z+\Delta z) / D}\right)}{\sigma\left(S_{z / D}\right) \sigma\left(S_{(z+\Delta z) / D}\right)}
$$
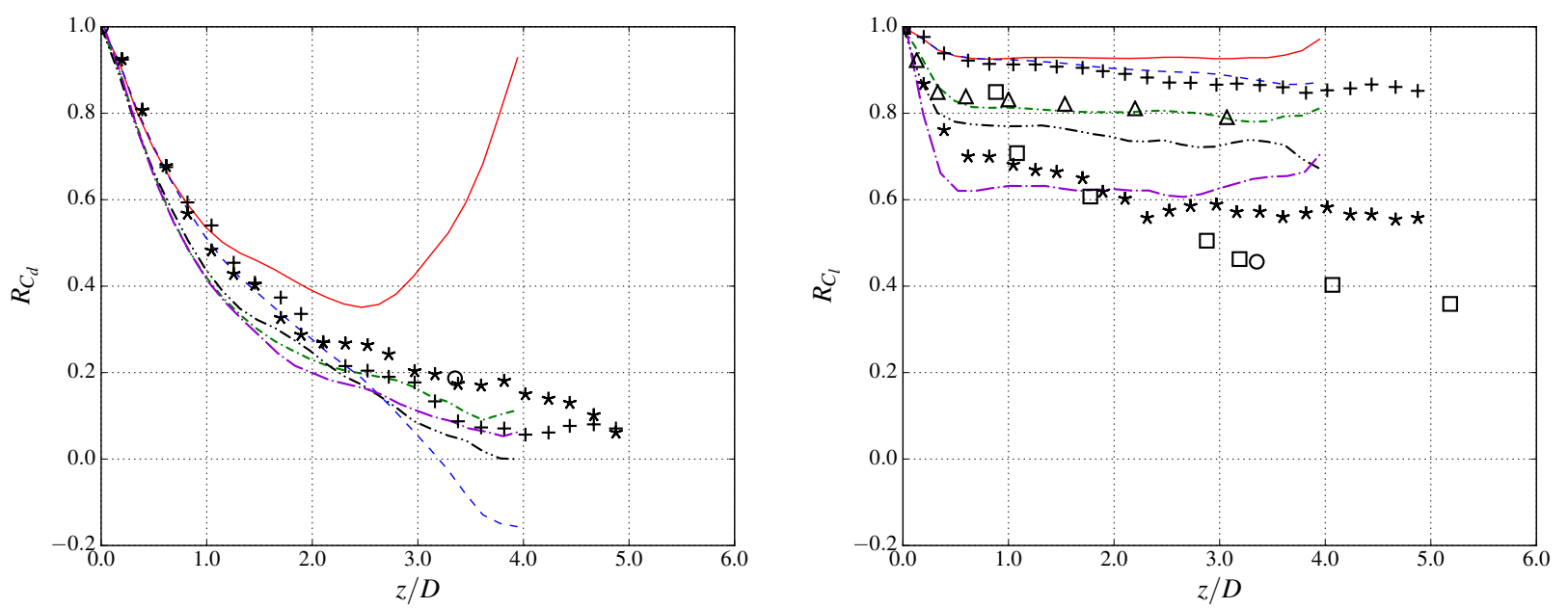

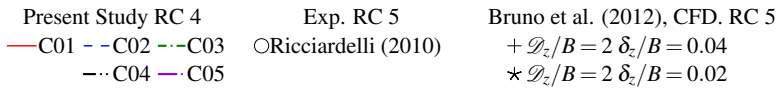

(a)
Present Study RC $4 \quad$ Experiments RC $5 \quad$ Bruno et al. (2012), CFD. RC 5 - C01--C02---C03 ORicciardelli (2010) $\quad+\mathscr{D}_{z} / B=2 \delta_{z} / B=0.04$ -.. C04-. C05 $\triangle$ Ito et al. (2014) $\quad \star \mathscr{D}_{z} / B=2 \delta_{z} / B=0.02$ $\square$ Li et al. (2016)

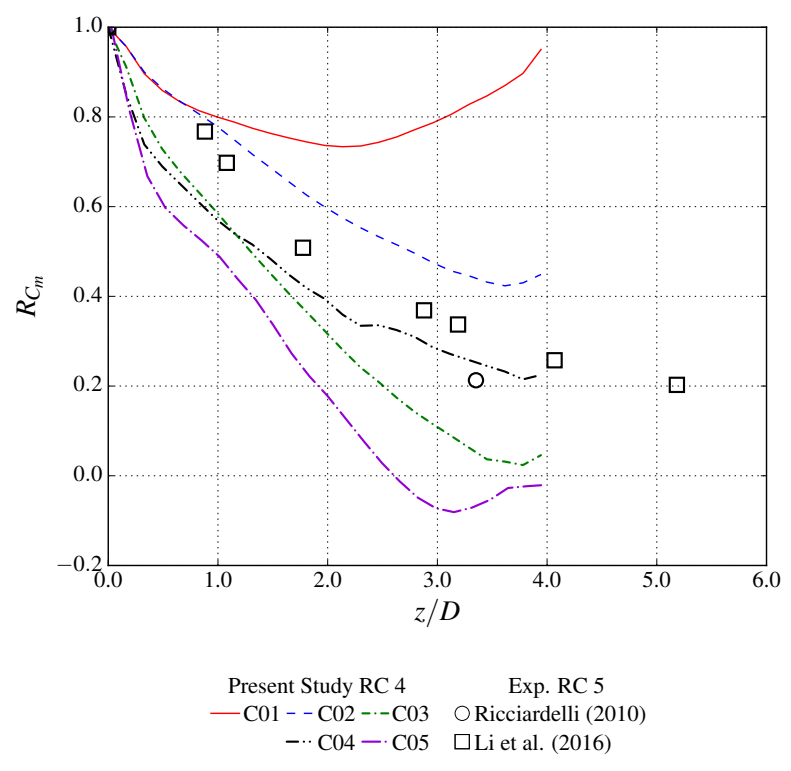

(c)

Figure 5: Spanwise correlation of (a) the Drag coefficient, (b) the Lift coefficient and (c) the Moment coefficient for the different cases studied. 
It has been found that the spanwise correlation of the drag coefficient is relatively insensitive to the mesh discretisation. On the other hand, the lift coefficient correlation decreases with the mesh resolution. A similar trend may be identified in the correlation of the moment coefficient, except for case C04 that provides higher correlation than the case $\mathrm{C} 03$ for $z / D>1.0$. It can also be appreciated that for the case C01 there is a recovery in the value of the spanwise correlation of the force coefficients, starting from the middle of the span approximately. This does not happen in the other cases studied, where a symmetric boundary condition is used.

The experimental data from Ricciardelli (2010) $\left(\mathrm{Re}=0.64 \cdot 10^{5}\right)$, Ito et al. $(2014)$, Li et al. $(2016)(\mathrm{Re}=$ $\left.0.46 \cdot 10^{5}\right)$, and the numerical results in Bruno et al. $(2012)\left(\operatorname{Re}=0.44 \cdot 10^{5}\right)$ for a 5:1 rectangular cylinder are included in figure 5, since to the authors' knowledge there are no available experimental data for the ratio $4: 1$ rectangular cylinder.

It has been found that the spanwise length in CFD models and its level of discretisation, play a very important role in obtaining correlation losses similar to the ones observed experimentally, as stated in Bruno et al. (2012), where the better agreements were found for the simulations with the longest spanwise distance and the finest of the discretisations.

Furthermore, the boundary conditions at the lateral sides yield different results. Previous simulations by other researchers, to the authors' knowledge, have been conducted using periodic boundary conditions in the lateral sides (see table 4), in which a recovery of the correlation values of the force coefficients is obtained for $\Delta_{z} \geq L / 2$, meanwhile the use of symmetric boundary conditions in these lateral sides, provides correlation values which do not present this issue along the spanwise length, as can be seen in figure 5. Furthermore, the numerical results are similar to the ones yielded by simulations adopting periodic boundary conditions with similar discretisation in the spanwise length, presenting longer distances in the spanwise direction.

\subsection{Main flow features, and mean and fluctuating pressure coefficient distributions}

In this section the main flow features are identified by means of studying streamlines, friction coefficient, and mean and fluctuating pressure coefficient distributions. The latter ones will be compared with available experimental data.

In figure 6 the time averaged and spanwise averaged streamlines for the cases C01 and C05 are presented, as the results yielded by the cases C02, C03 and C04 are very similar. All the cases show the same flow regions, which have been identified as: inner region, recirculation region, main vortex and reattachment. These regions are the same as the ones described in Bruno et al. (2010), for the 5:1 rectangular cylinder, which is characterised by a permanently reattached flow (Bruno et al., 2014), as the 4:1 rectangular cylinder considered herein.

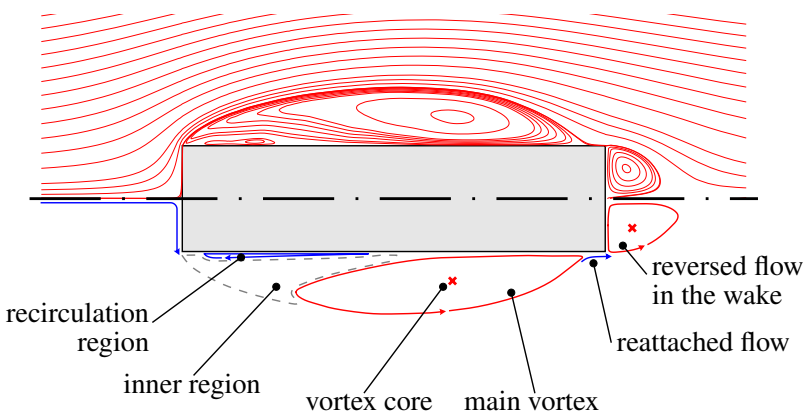

(a) $\mathrm{C} 01$

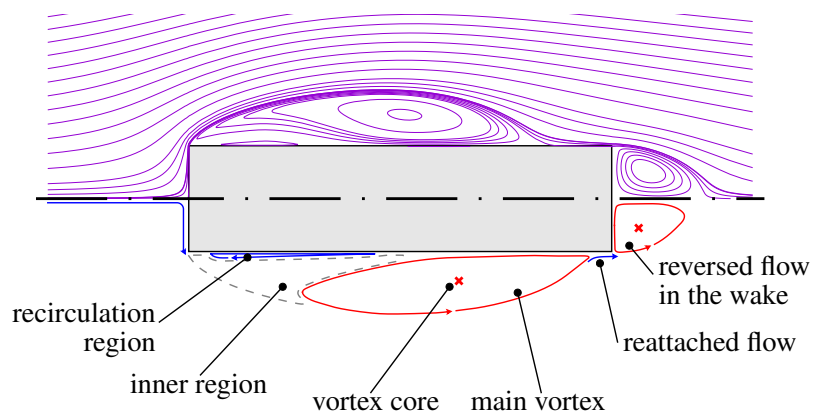

(b) $\mathrm{C} 05$

Figure 6: Time averaged and spanwise averaged streamlines for the different cases (upper half). Recognised mean flow structures (lower half). 
In figure 7 , the time averaged, spanwise averaged and side averaged friction coefficient $\left(C_{f}\right)$ distribution is presented for the different cases. The length of each region, previously identified, is given by the sign change of the friction coefficient. The lengths of the different regions for the different cases are presented in table 7 . As the refinement of the meshes increases the reattachment takes place further away from the leeward edge and therefore the reattachment length $\left(l_{r e a}\right)$ increases. The lengths corresponding with the main vortex $\left(l_{m v}\right)$ and the inner region $\left(l_{i n}\right)$ are kept fairly constant. However, the recirculation region shrinks as the spatial discretisation increases, which is apparent from the decrease in the length of the recirculation region $\left(l_{\text {rec }}\right)$, according to the data in table 7 .

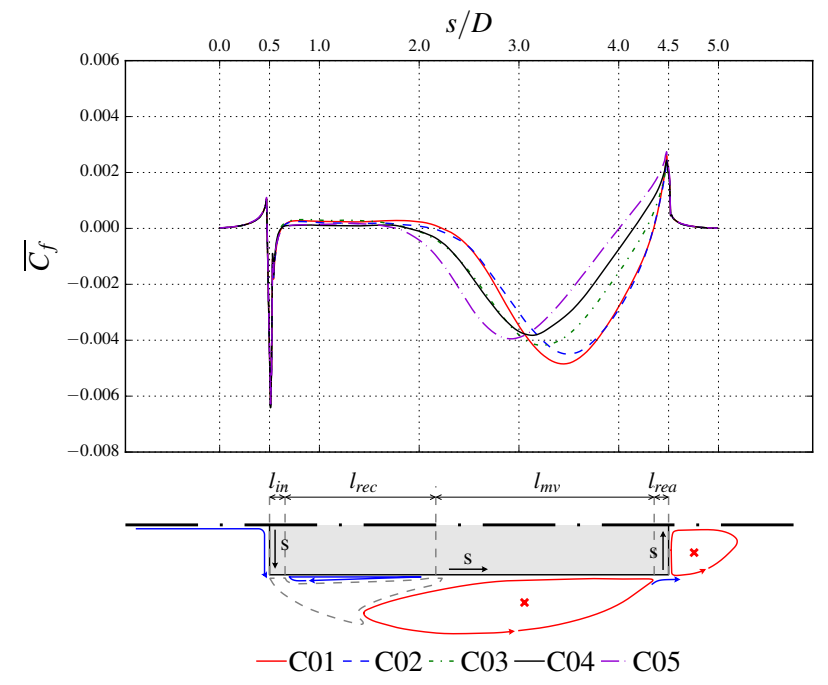

Figure 7: Time averaged, spanwise averaged and side averaged friction coefficient distributions for the different cases (upper half). Recognised mean flow structures(lower half).

Table 7: Length of the different fluid structures.

\begin{tabular}{ccccc}
\hline Case & $l_{\text {in }} / D$ & $l_{\text {rec }} / D$ & $l_{m v} / D$ & $l_{\text {rea }} / D$ \\
\hline $\mathrm{C} 01$ & 0.14 & 1.56 & 2.15 & 0.15 \\
$\mathrm{C} 02$ & 0.11 & 1.50 & 2.24 & 0.15 \\
$\mathrm{C} 03$ & 0.10 & 1.30 & 2.37 & 0.23 \\
$\mathrm{C} 04$ & 0.11 & 1.29 & 2.25 & 0.35 \\
$\mathrm{C} 05$ & 0.14 & 1.15 & 2.21 & 0.5 \\
\hline
\end{tabular}


Finally, in figure 8 the mean and standard deviation pressure coefficient distributions are compared with available experimental data, showing a good agreement between the experimental and numerical data, particularly as the mesh is progressively refined. The numerical results show certain sensitivity to the overall number of elements, creating an earlier pressure recovery and reducing the standard deviation of the pressure coefficient $\left(\widetilde{C_{p}}\right)$ as the number of elements increases. In the pressure recovery region, the changes in the mean and fluctuating pressure were smooth as the meshes were refined.

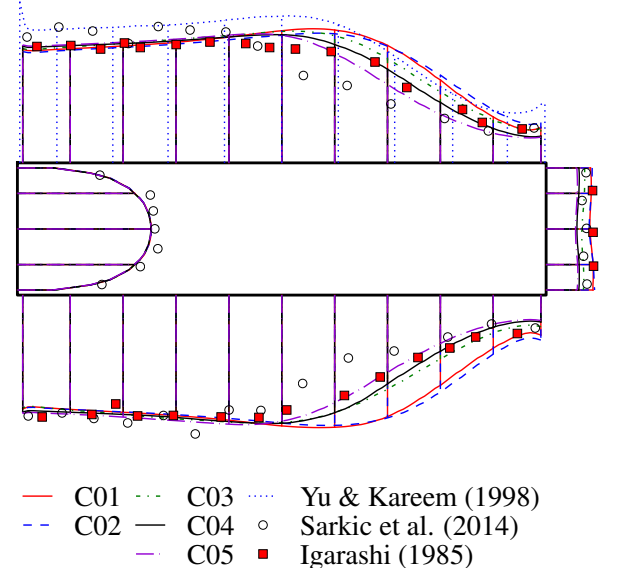

(a)

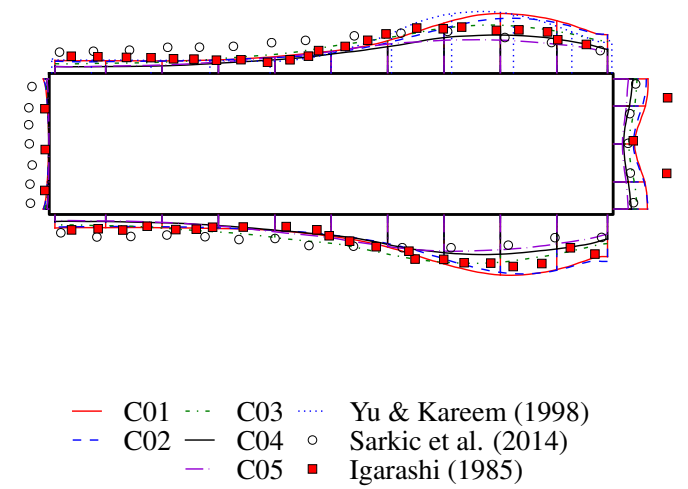

(b)

Figure 8: (a) Pressure coefficient mean distribution $\overline{C_{p}}$. (Negative values point outwards and the value of unity is equal to the cylinder height) (b) Pressure coefficient standard deviation distribution $\widetilde{C_{p}}$ (Positive values point outwards and the value of unity is equal to the cylinder height).

\subsection{Pressure correlation distribution and coherence}

In figure 9 the side averaged spanwise surface pressure correlations for the different cases are presented. It is again clearly appreciated the effect of the periodic boundary condition on the spanwise correlation. As shown in the case C01 (figure 9a), a recovery in the correlation starts approximately from the middle of the span, as reported in the correlation of the force coefficients (figure 5).

In addition, the lengths of the mean flow structures identified in section 3.2 are included in the pressure correlation distribution charts, aiming to better understand the physical significance of the reported results. It has been found that the side facing the incoming flow presents the highest values of the correlation and they smoothly decrease as the spanwise distance increases. The pressure correlation over the horizontal side length near the windward corner, corresponding with the tip of the inner region, shows relatively high values of pressure correlation, increasing smoothly towards the recirculation region. The correlation strongly diminishes in the recirculation region as the distance from the windward corner and the spanwise length increase. In fact, the lowest values in pressure correlation are found along the boundary between the recirculation and the main vortex region, which may be explained by the complex interaction that takes place between the tail of the recirculation vortex and the main vortex above. This means that the flow in the recirculation region is disorganised as it progresses towards the boundary with the main vortex region. In a similar fashion, the flow moving in the main vortex region also becomes more highly correlated as it progresses downstream. The flow structure is once more disorganized as the reattachment region is approached by the flow, even though the correlations are higher than in the interface between the recirculation and the main vortex regions. Finally, it is identified an increase in correlation over the leeward side of the cylinder as a reversed flow vortex in created is the wake (see figure 6).

The five cases provide the same qualitative description of the pressure correlations over the surface of the cylinder. However, as the spatial discretisation is improved, faster changes in the correlation values are identified and the portion of the surface showing correlations above 0.3 clearly disminishes. 


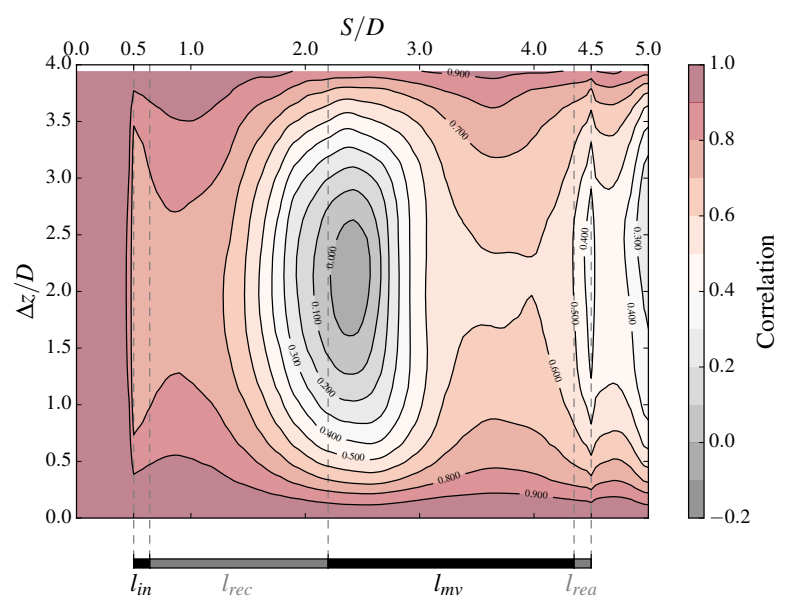

(a) $\mathrm{C} 01$

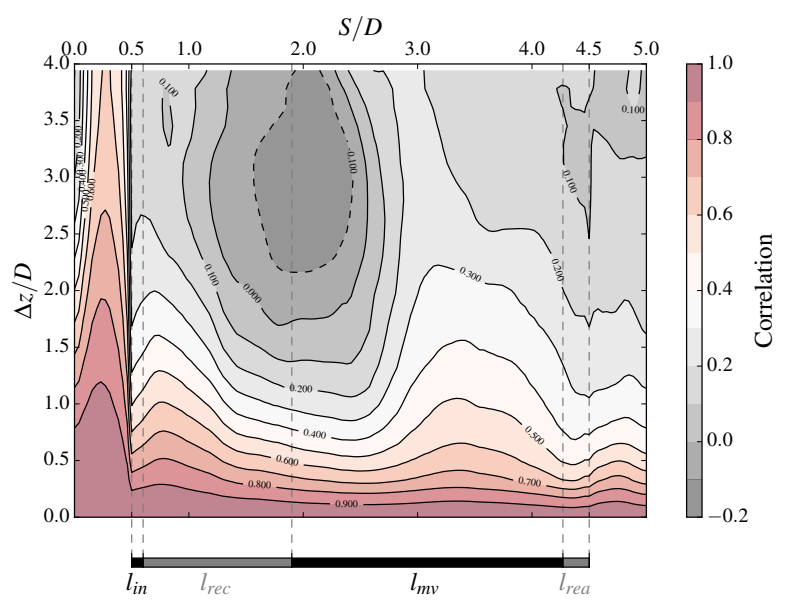

(c) $\mathrm{C} 03$

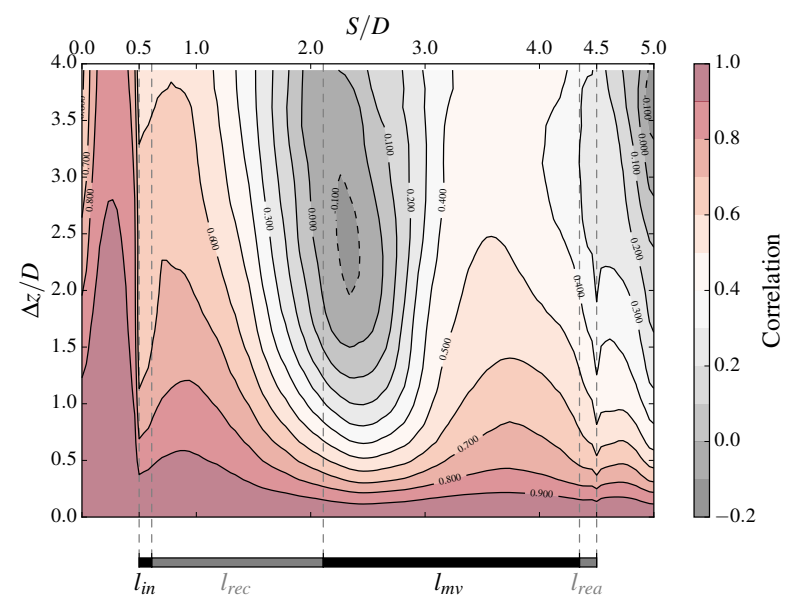

(b) $\mathrm{C} 02$

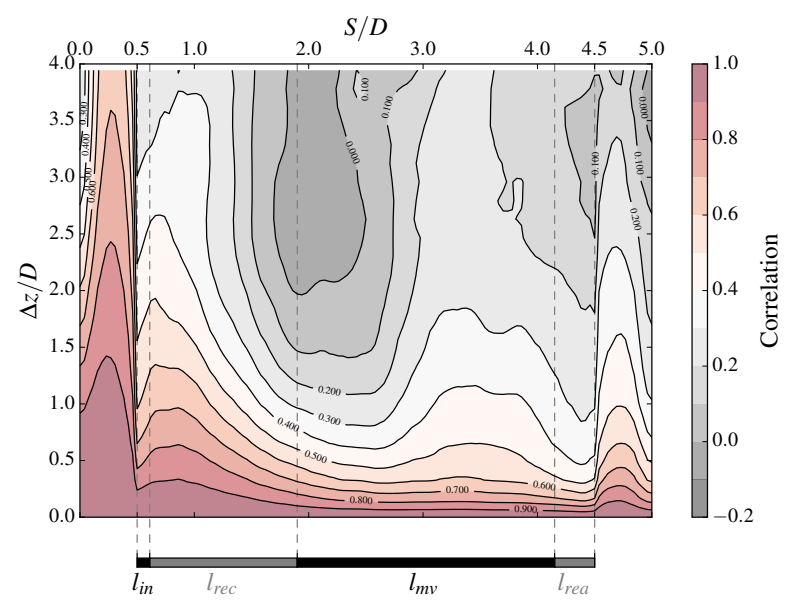

(d) $\mathrm{C} 04$

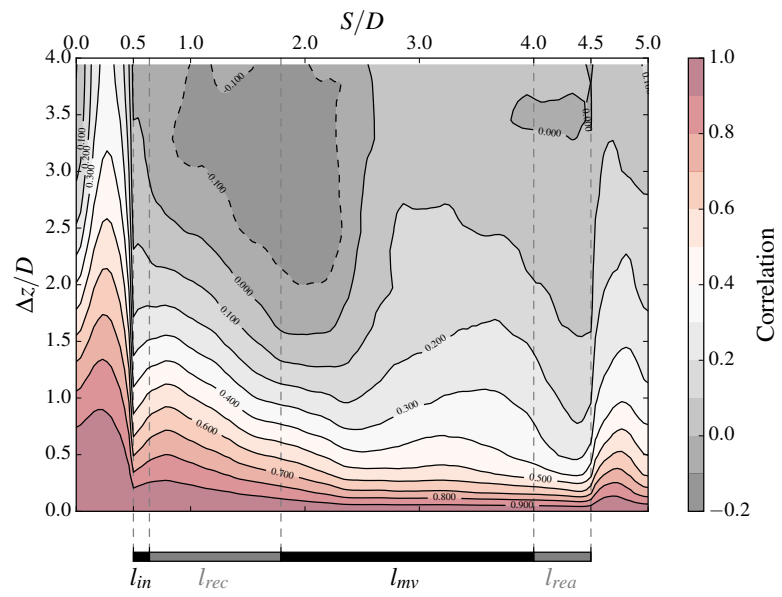

(e) $\mathrm{C} 05$

Figure 9: Pressure correlation distributions for the different cases. 
In order to further study the existence of coherent structures along the spanwise direction, the pressure coherence is calculated at two different $x$ positions. For each one of these positions two different spanwise distances have been considered. The reference point in $z(z=0)$ is the middle of the spanwise dimension. The coherence is calculated as expressed in equation (11), where, $S_{z=0}(p)$ is the power spectrum of the pressure time-history located at the reference point, $S_{z=\Delta z}(p)$ is the power spectrum of the pressure time-history separated $\Delta z$ from the reference point and $S(\Delta z ; p)$ is the cross-spectrum between the pressure time-histories from where the power spectra were calculated.

$$
\operatorname{Coherence}(\Delta z ; p)=\frac{|S(\Delta z ; p)|}{\sqrt{S_{z=0}(p)} \sqrt{S_{z=\Delta z}(p)}} .
$$

In figure 10, the positions at which the power spectrum densities of the pressure are obtained, are shown for the five studied cases. Meanwhile, in figure 11, the coherences of the points presented in figure 10 are reported and compared with the experimental results obtained by (Matsumoto et al., 2003) for a ratio 5 rectangular cylinder, since to the authors' knowledge there are not available data in the literature for the 4:1 ratio. For comparison purposes, as the Strouhal number of the ratios 4 and 5 cylinders are different, the horizontal axis variable containing the non dimensional frequency is divided by their corresponding St. The maximum coherence peak may be identified at the $S t$ value, for both the experimental and numerical time-histories. At both sides of the $S t$ the coherence decreases. This phenomenon takes place in all cases, reducing the magnitude of peaks as the mesh is progressively refined. This refinement also causes the reduction in the coherence peak value around the $S t$. It may be also appreciated how the coherence in the vicinity of $S t$ decreases more intensely in the proximity of the leeward side $(x / D=1.6)$. Similarly, for larger spanwise distances $\left(\Delta_{z} / D=1.0\right)$, the coherence is lower around $((f D) / U) / S t=1$. The high coherence region around the $S t$ is wider in the numerical cases than in the experimental case, and similar results are obtained by (Bruno et al., 2010) for a 3D LES simulation of a ratio 5 rectangular cylinder. The lower resolution appreciated in the numerical coherences is due to the sampling time, lower than in the experimental case.

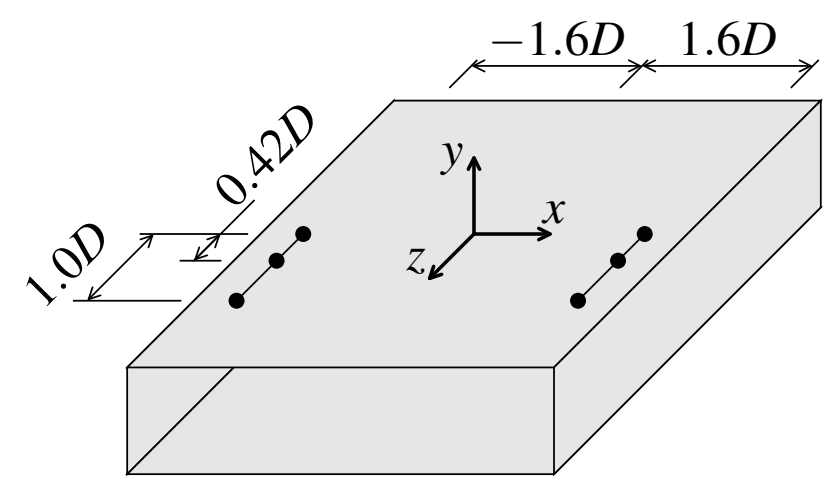

Figure 10: Location for the calculation of the coherence.

Also, in figure 12, the comparison of the power spectrum densities of the pressure for both $x$ strips, at a spanwise position $z=0.0$ and $z=0.42 D$ (see figure 10) for cases C01 and C05 are shown. It may be observed how the signal components are concentrated at the Strouhal number in the case C01, with a lower spatial discretisation. As the discretisation level is augmented, the energy content is spread over different frequency components. In case C05, although the main peak of the power spectrum density does not coincide with the Strouhal number, also an important part of the energy is concentrated at $S t$ for the two positions shown (figures 12c and 12d). This explains the high coherence values in figure 11, as coherence, in accordance with Xu (2013), "is approximately equivalent to a frequency-dependent correlation coefficient". Therefore, it allows to compare how similar are the different frequency components of the considered signals. 


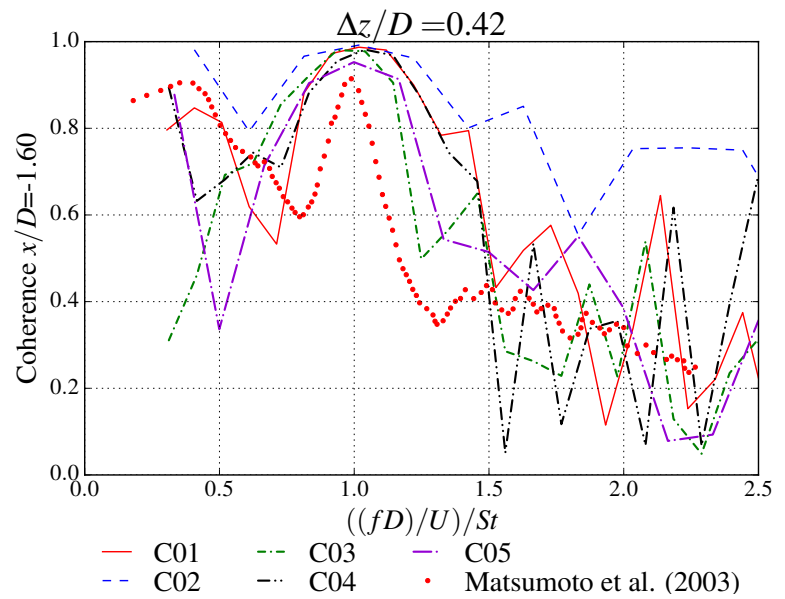

(a)

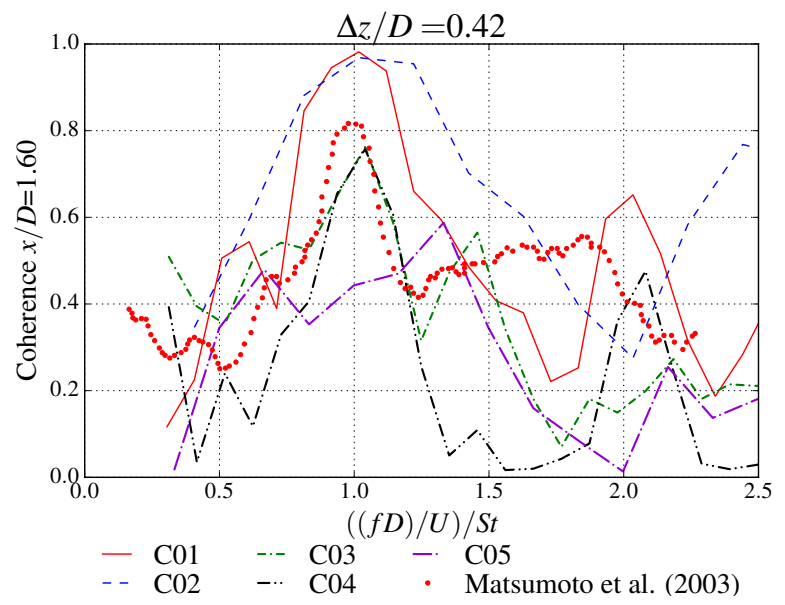

(c)

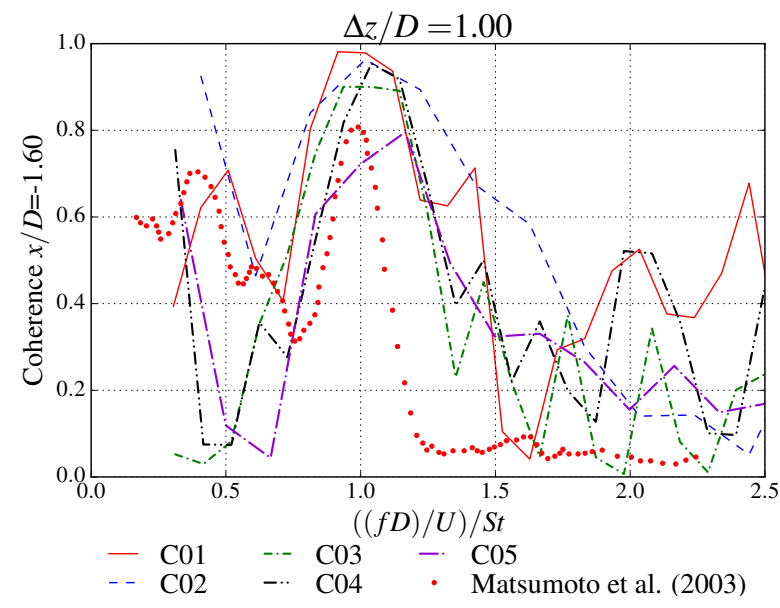

(b)

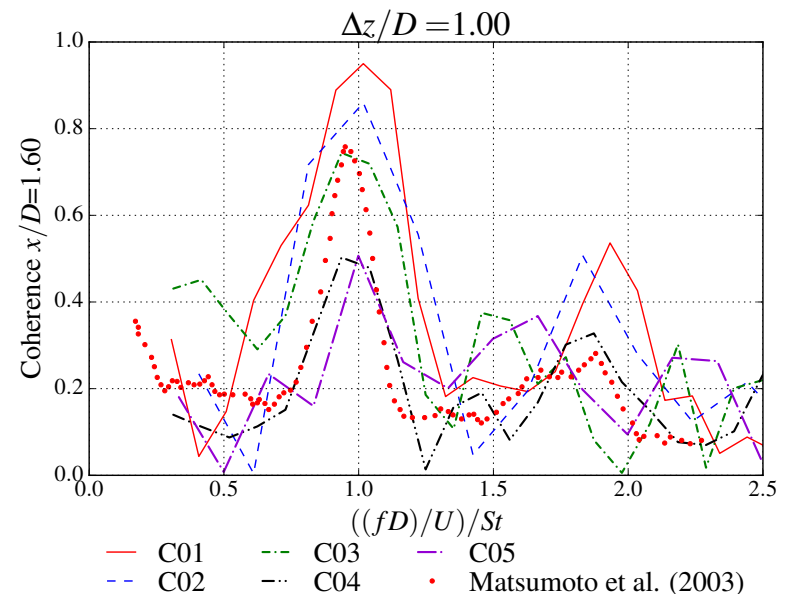

(d)

Figure 11: Coherence at different positions for the different cases. (a) $x / D=-1.60 \Delta_{z} / D=0.42$, (b) $x / D=-1.60 \Delta_{z} / D=1.00$, (c) $x / D=1.60 \Delta_{z} / D=0.42$ and (d) $x / D=1.60 \Delta_{z} / D=1.00$ 


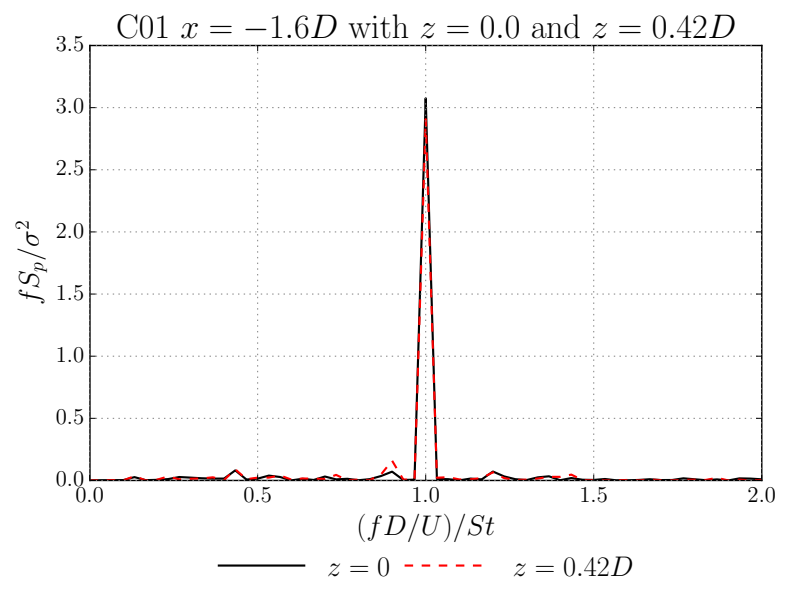

(a)

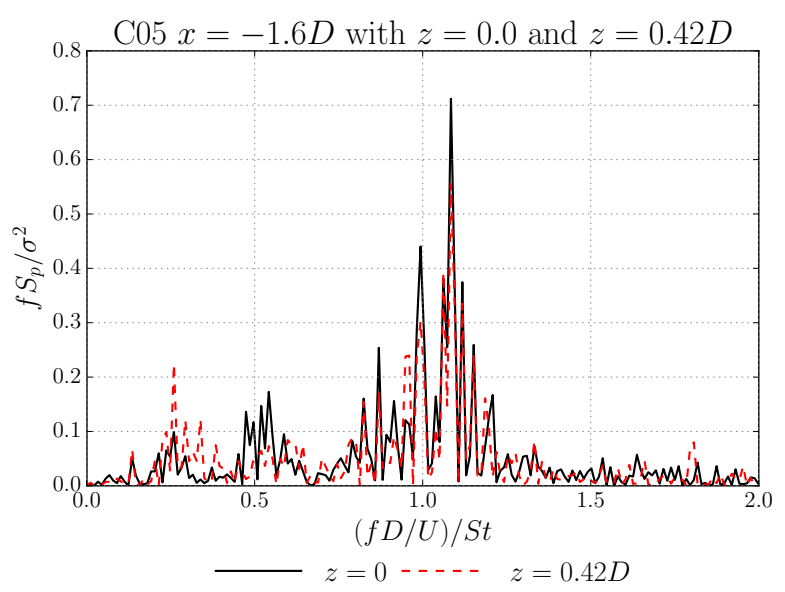

(c)

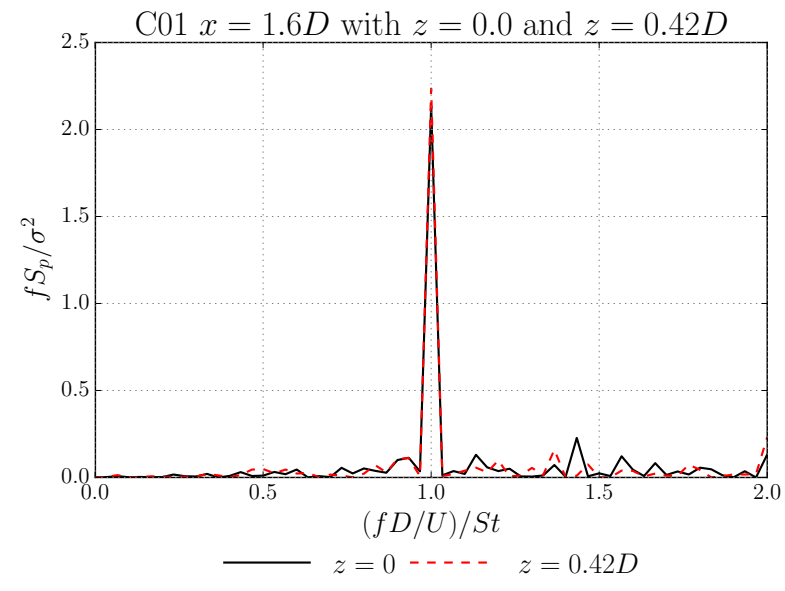

(b)

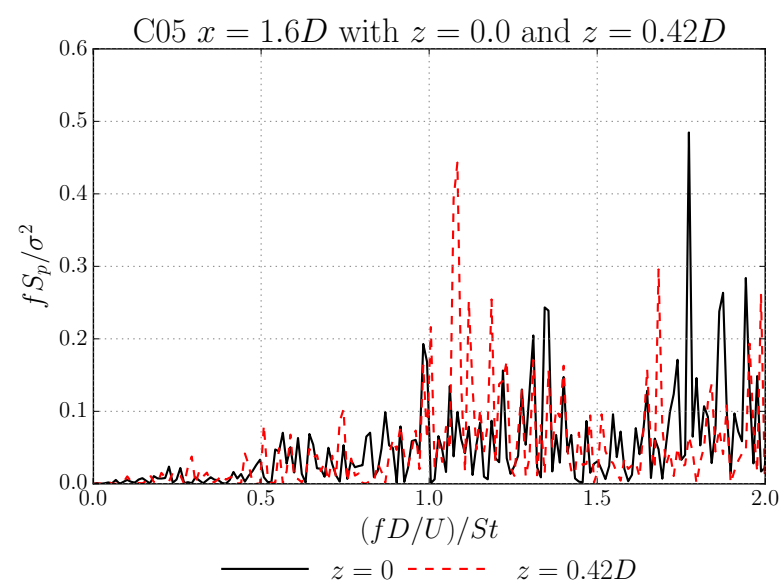

(d)

Figure 12: Power spectrum densities at different locations for cases C01 and C05. 


\section{Results for the free-to-oscillate case}

The simulations for the vertically free-to-oscillate rectangular cylinder were performed for a Scruton number of $6.0(S c=(4 \pi M \xi) /(\rho B D L)$, where $M$ is the mass of the cylinder, $\xi$ is the relative damping, $\rho$ is the air density and $L$ is the spanwise dimension). The Scruton number is the same as in the experiments carried out by Marra et al. (2011), which are adopted here for validation. Meshes in cases C03, C04 and C05 were selected to conduct the VIV simulations since lower resolution meshes provided poorer agreement with experimental data for the static case. The simulations conducted in this section have Reynolds numbers ranging from $0.3 \cdot 10^{5}$ to $0.55 \cdot 10^{5}$. It can be seen in figure 13 a that the finer the discretisation, both in the spanwise dimension and in the $X Y$ plane, the better the computed maximum structural response in comparison with the experimental values in Marra et al. (2011) and the numerical simulations in Daniels et al. (2016). This can be explained by a reduction in the spanwise correlation of the lift coefficient as the spatial discretisation was improved, as reported in the static simulation (figure 5b), as well as the higher pressure fluctuations observed in the coarser meshes (figure 8). The on-set reduced velocity of the VIV slightly varied with respect to the experimental data, and this can be explained by the subtle differences in the value of the Strouhal number between the experimental case and the simulations. The mesh refinement in the spanwise dimension provides the lock-in range closer to the experimental ones. Also, for all the cases, the fluctuation of the structural response and lift force is locked into the natural frequency of the model $\left(f_{0}\right)$ during the VIV lock-in. After this region, the heave oscillation frequency remains equal to the system frequency, meanwhile the lift force frequency follows the vortex shedding frequency defined by the Strouhal number (see figure 13b). The termination of the lock-in region coincides with the instance when the lift force and vertical displacement phase lag angle is around $180^{\circ}$ (see figure $13 \mathrm{c}$ ) (Nguyen et al., 2018). Therefore it is highlighted that the accurate simulation of the motion induced forces is linked with the level of discretisation in the spanwise dimension and the ability of the model to properly simulate the correlation of aerodynamic forces along the spanwise dimension and the phase lag between lift force and heave oscillation.

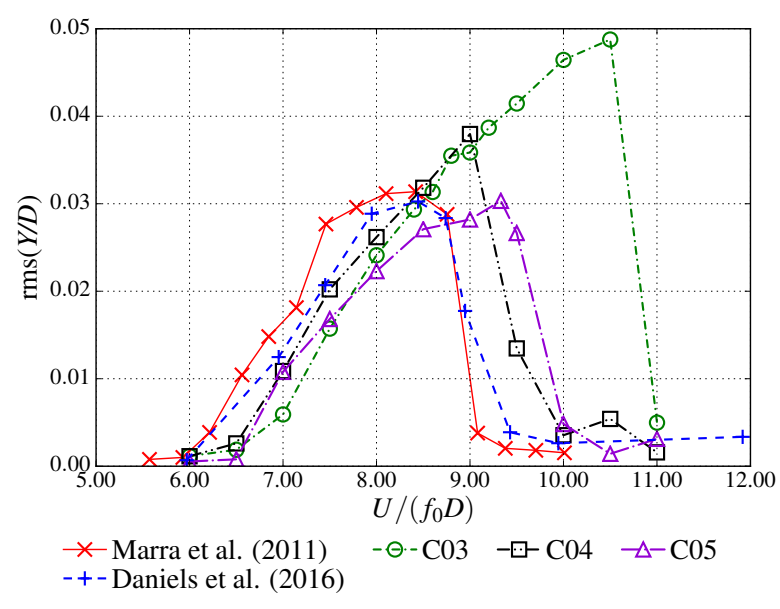

(a)

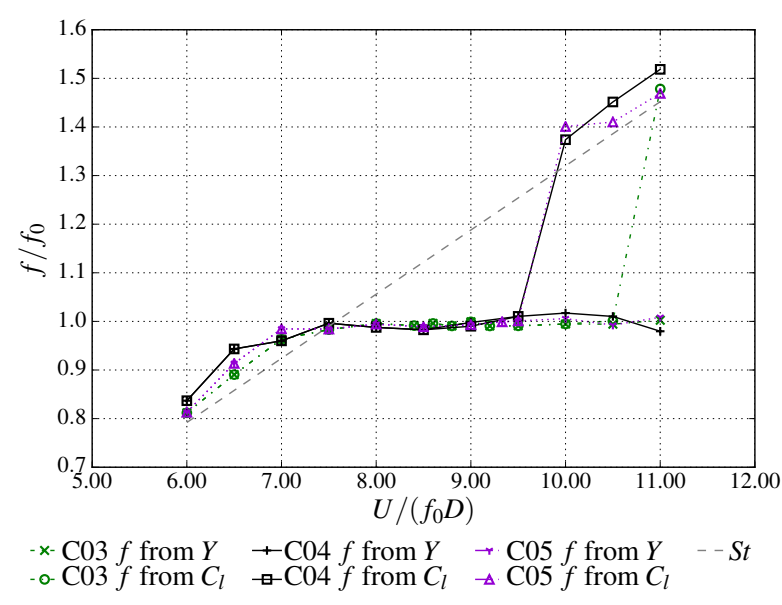

(b)

Figure 13: (a) Vibration amplitude, (b) Vibration frequency, (c) Phase lag between the oscillation in the heave degree of freedom and the $C_{l}$. 


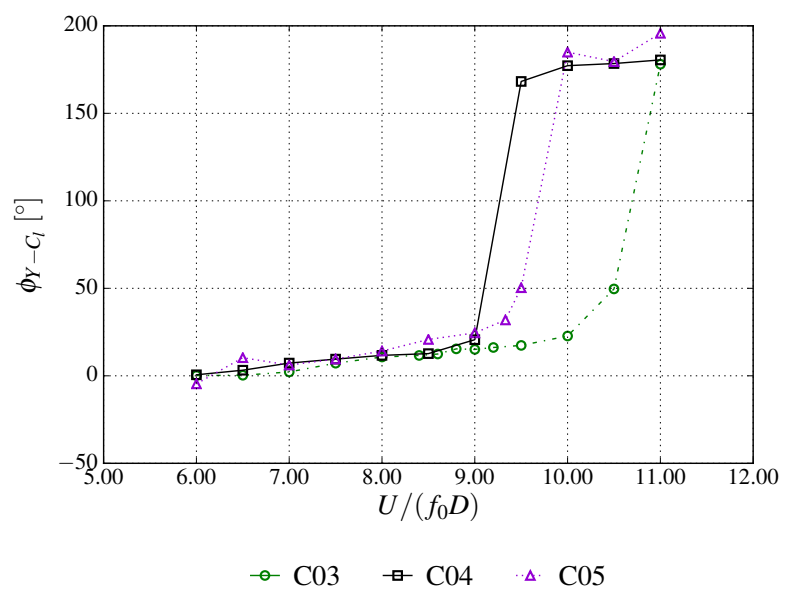

(c)

Figure 13: (a) Vibration amplitude, (b) Vibration frequency, (c) Phase lag between the oscillation in the heave degree of freedom and the $C_{l}$. [Cont.]

\subsection{Phase averaged pressure coefficient distribution}

In order to obtain more information about the impact of the mesh discretisation level over VIV, in figure 14 the heave displacement and the corresponding phase averaged lift coefficient are presented, showing the phase lag between the two phase-averaged responses at the maximum VIV structural responses for cases C03, C04 and C05.

In figure 15, the phase averaged pressure coefficient distributions at 8 different positions along the heaving cycle at the reduced velocity for which the cylinder exhibits the maximum VIV structural response (see figure 13a) are reported.

The phased averaged pressure coefficient distributions at instants P01 to P04 have their corresponding mirrored pressure distribution in times P05 to P08 respectively. The high suction peaks along the top and bottom surface are related to the movement of the vortex travelling towards the leeward edge. The generation of the vortices from the windward edge is synchronized with the oscillation of the cylinder, keeping on growing and travelling for a cycle, and then being finally shed from the leeward side (Komatsu and Kobayashi, 1980). The generation and shedding of the vortex located at the upper wall takes place at the instant "P01" whilst in the case of the lower wall this happens at the instant "P05", which gives the characteristic alternation of the vortex shedding for this geometric configuration.

It is also appreciated in figure 15, that with finer mesh resolutions, the pressures on the leeward half of the upper and lower surfaces of the cylinder, experiment smaller fluctuations along the oscillation cycle, which also helps to explain lower amplitudes in the VIV response as the grid is progressively refined. 


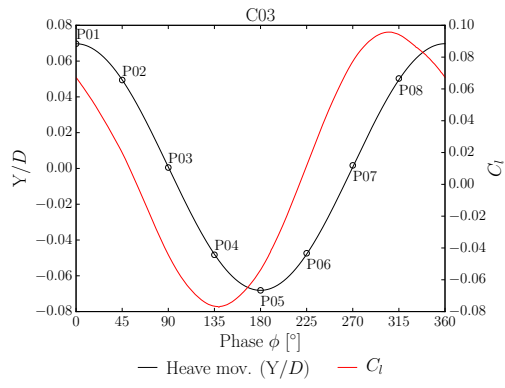

(a)

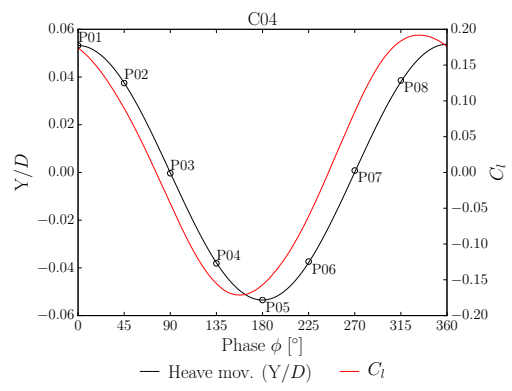

(b)

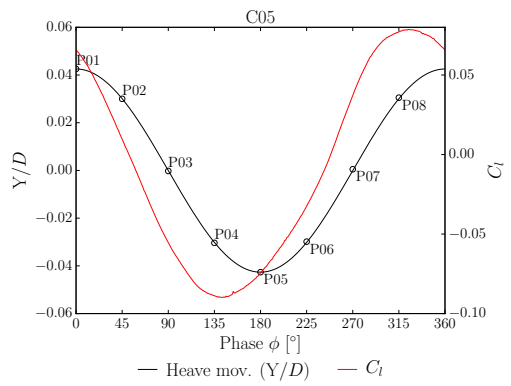

(c)

Figure 14: Heave movement cycle and phase averaged $C_{l}$ for the cases (a) C03 $\left(U_{r}=10.5\right)$, (b) $\mathrm{C} 04\left(U_{r}=9.0\right)$ and (c) $\mathrm{C} 05\left(U_{r}=9.3\right)$
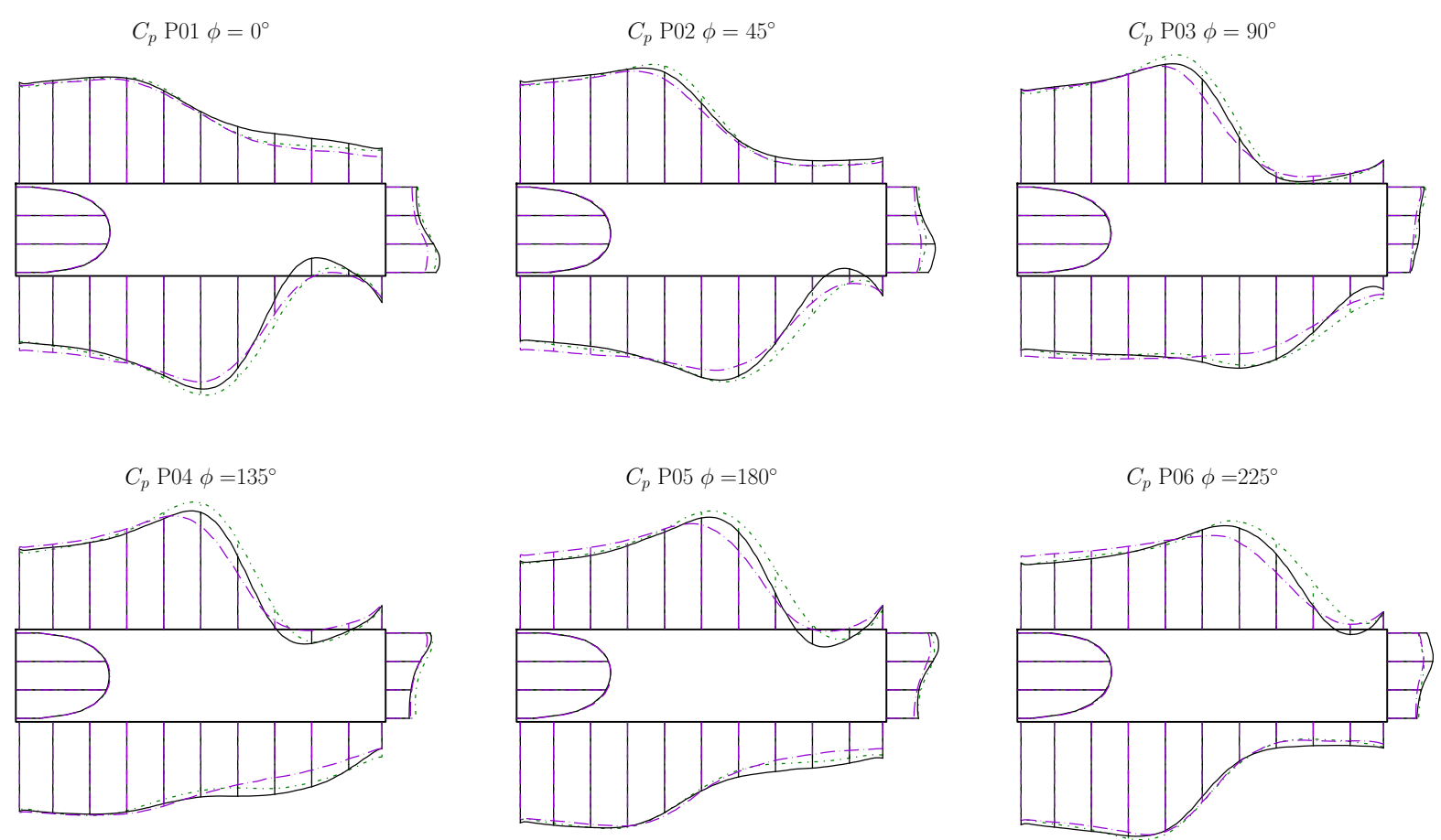

$C_{p} \mathrm{P} 07 \phi=270^{\circ}$

$C_{p} \mathrm{P} 08 \phi=315^{\circ}$
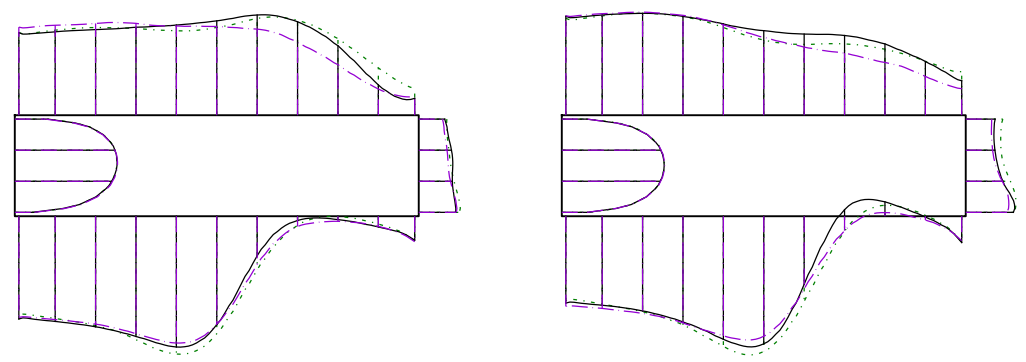

-. $\mathrm{C} 03-\mathrm{C} 04-\mathrm{C} 05$

Figure 15: Phase averaged pressure distribution for the cases $\mathrm{C} 03\left(U_{r}=10.5\right), \mathrm{C} 04\left(U_{r}=9.0\right)$ and $\mathrm{C} 05$ $\left(U_{r}=9.3\right)$ at maximum response flow velocity. (Negative values points outwards and the value of unity is equal to the cylinder height) 


\subsection{Pressure coefficient correlations}

In figure 16, the spanwise correlations of pressure on the surfaces of the upper half of the cylinder, when it experiences the maximum VIV structural response for cases C03, C04 and C05 are presented. They are in good agreement with the experimental data for the 5:1 rectangular cylinder reported in Nguyen et al. (2018), since to the authors knowledge, equivalent experimental data for the 4:1 rectangular cylinder are not available in the literature. The spanwise correlation at the points closer to the windward edge presents in general higher values than in the locations closer to the leeward edge, and in all the cases studied, the correlations are higher than the ones obtained for the static case. This is in agreement with the results obtained from experimental campaigns for the square cylinder (Wilkinson, 1981) and the ratio 5 rectangular cylinder (Ricciardelli, 2010; Li et al., 2016) that have identified higher correlations of aerodynamic forces for oscillating bodies. In figure 16, in all the cases, two zones with high correlations are identified on the upper surface, in the vicinity of the windward and leeward corners. Over the central part of the upper side, lower pressure coefficients correlations are obtained. The high correlation zone close to the windward corner is associated to the motion-induced vortex generated at the leading edge, described in Matsumoto et al. (2008). This vortex travels on the body surface, developing three-dimensional features and giving place to more uncorrelated flow structures, explaining the correlation loss in the middle part of the upper surface. Finally, this vortex coalesces with the secondary vortex that is generated at the trailing edge when it reaches this position, explaining the recovery in the pressure correlations identified when approaching to the leeward corner. No clear trend in the values of the pressure correlations is identified as the grid is progressively refined, although case C05 provides lower magnitudes as the spanwise distance increases, that are in better agreement with the experimental values in Nguyen et al. (2018). It may be argued that this is due to the fact that the maximum response corresponds to different reduced velocities for cases C03, C04 and C05.

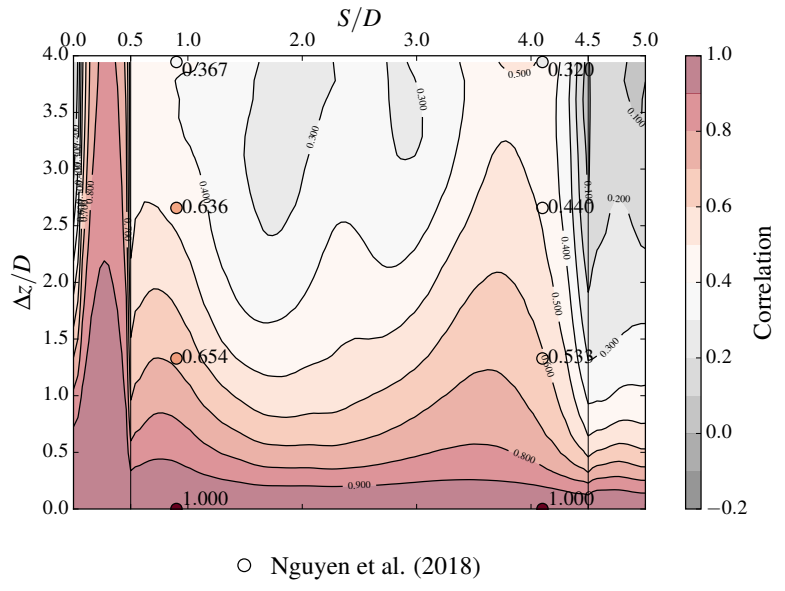

(a) $\mathrm{C} 03$

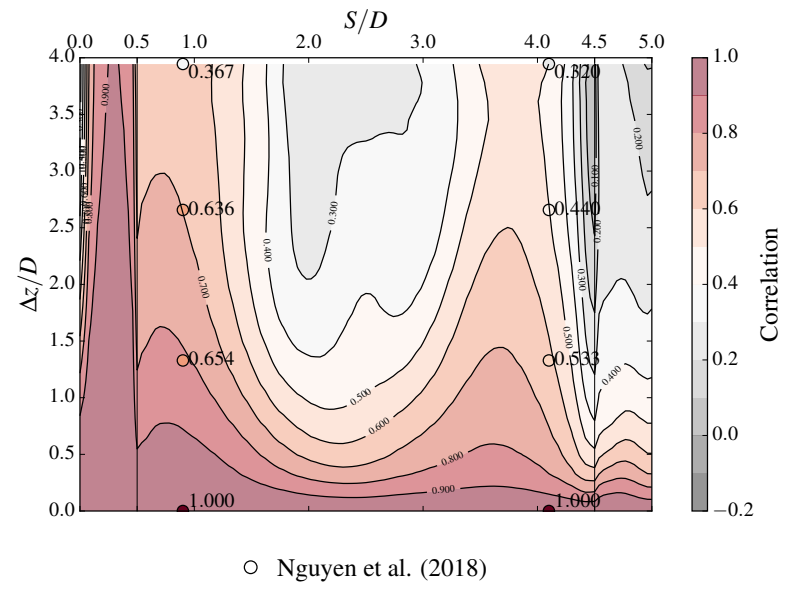

(b) $\mathrm{C} 04$

Figure 16: VIV heave pressure correlation surfaces for the cases C03, C04 and C05 at the point with the maximum response amplitude. 


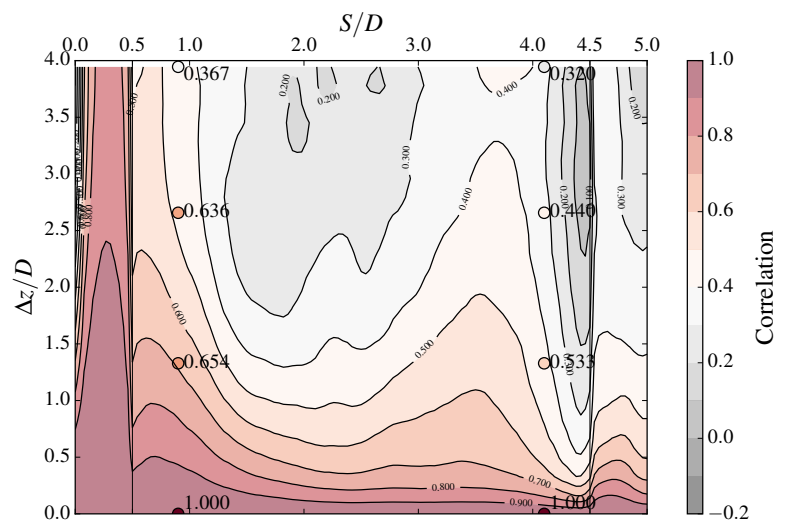

○ Nguyen et al. (2018)

(c) $\mathrm{C} 05$

Figure 16: VIV heave pressure correlation surfaces for the cases C03, C04 and C05 at the point with the maximum response amplitude. [Cont.]

Despite the fact of using shorter spanwise dimensions in the current simulations than in previous numerical investigations of rectangular cylinders undergoing VIV heave oscillations (Daniels et al., 2016; Nguyen et al., 2018), the use of symmetric boundary conditions at the lateral sides of the flow domain, in combination with a sufficient level of discretisation, has provided correlations and amplitudes of oscillation in agreement with experimental data. Therefore, the selection of symmetric boundary conditions are allowing a reduction of the domain length in the spanwise dimension.

\section{Conclusions}

Numerical simulations for the static 4:1 rectangular cylinder have been conducted for different types of boundary conditions and mesh discretisations. It has been found that the application of the symmetric boundary condition on the lateral surfaces of the fluid domain provides a more physically sound spanwise correlation.

All the simulations of the static 4:1 rectangular cylinder provide force coefficients, Strouhal number and pressure coefficient distributions in good agreement with the available experimental data. No major differences appear in the force coefficients and Strouhal number when the different mesh discretisations are applied, except for the variations in the standard deviation of the pressure coefficient distributions and force coefficients. The simulations are able to identify different flow structures developing over the cylinder; also the size of each of them was calculated. The pressure coherence at different locations was calculated and compared with experimental data, finding a general good agreement with the 5:1 rectangular cylinder. The pressure correlation surfaces were obtained, and helped to understand the flow characteristics in the identified flow regions.

On the other hand, the vertically free-to-oscillate simulations present a dramatic sensitivity with the spanwise discretisation and the overall number of elements in the $X Y$ plane; the VIV lock-in interval is better estimated by using the mesh having a finer discretisation in the spanwise dimension, meanwhile the maximum structural response during the lock-in is better approximated when the mesh is further refined in both the spanwise dimension and the $X Y$ plane. Therefore, to correctly simulate the motion induced forces, using a fine enough discretisation along the spanwise dimension as well as in the $X Y$ plane is one of the key requirements in 3D LES modelling, finding that a feasible dimension in the spanwise direction is $\delta_{z} / B=0.02$. Although for higher levels of turbulence in the incoming flow or higher heave oscillation amplitudes the discretisation requirements in the spanwise direction may be different. For the cases considered, phase-averaged distribution of $C_{p}$ as well as the spanwise correlation of pressure at different locations were calculated as the maximum VIV structural response is observed. These correlations were compared with experimental data and were found to be in good agreement with them. 


\section{Acknowledgements}

This research has been funded by the Spanish Ministry of Economy and Competitiveness in the frame of the research project with reference BIA2016-76656-R and the Galician regional government with reference ED431C 2017/72. The first of the authors has been funded by the Spanish Ministry of Economy and Competitiveness in the frame of the National Program for Promotion of Talent and Employability through the BES-2014-068418 predoctoral contract grant, associated with the BIA2013-41965-P research project and the EEBB-I-17-11905 international exchange programme. The advice, valuable comments and support of Prof. David Hargreaves is greatly appreciated.

The computations have been carried out in the computer cluster Breogan and in the Galician Supercomputation Technology Centre (CESGA).

The authors fully acknowledge the received support.

\section{References}

Arslan, T., Petterson, B., and Anderson, H. I. (2011). Calculations of the flow around rectangular shaped floating structures. In Proceedings of 13th Internatinal Conference on Wind Engineering, Amsterdam, The Netherlands.

Bruno, L., Coste, N., and Fransos, D. (2012). Simulated flow around a rectangular 5:1 cylinder: Spanwise discretisation effects and emerging flow features. Journal of Wind Engineering and Industrial Aerodynamics, 104-106:203-215.

Bruno, L., Fransos, D., Coste, N., and Bosco, A. (2010). 3d flow around a rectangular cylinder: A computational study. Journal of Wind Engineering and Industrial Aerodynamics, 98:263-276.

Bruno, L., Salvetti, M. V., and Ricciardelli, F. (2014). Benchmark on the aerodynamics of a rectangular 5:1 cylinder: An overview after the first four years of activity. Journal of Wind Engineering and Industrial Aerodynamics, 126:87-106.

Daniels, S. J., Castro, I. P., and Xie, Z. (2016). Numerical analysis of freestream turbulence effects on the vortexinduced vibrations of a rectangular cylinder. Journal of Wind Engineering and Industrial Aerodynamics, 153:13-25.

de Villiers, E. (2006). The potential of large eddy simulation for the modeling of wall bounded flows. PhD thesis, Imperial College of Science, Technology and Medicine, Department of Mechanical Engineering, Thermofluids Section, London, UK.

Grozescu, A. N., Bruno, L., Fransos, D., and Salvetti, M. V. (2011a). Large-eddy simulations of a benchmark on the aerodynamics of a rectangular 5:1 cylinder. In Atti del 20. congresso dell'Associazione Italiana di Meccanica Teorica e Applicata, Bologna, Italy.

Grozescu, A. N., Salvetti, M. V., Camarri, S., and Buresti, G. (2011b). Variational multiscale large-eddy simulations of the barc flow configuration. In Proceedings of 13th Internatinal Conference on Wind Engineering, Amsterdam, The Netherlands.

Igarashi, T. (1985). Characteristics of the flow around rectangular cylinders. Bulletin of the Japan Society of Mechanical Engineers, 28(242):1690-1696.

Ito, Y., Shirato, H., and Matsumoto, M. (2014). Research on span-wise correlations of fluctuating lift forces on rectangular cylinders with various side ratio. In Proceedings of the 6th International Symposium on Computational Wind Engineering, Hamburg, Germany.

Komatsu, S. and Kobayashi, H. (1980). Vortex-induced oscillation of bluff cylinders. Journal of Wind Engineering and Industrial Aerodynamics, 6:335-362. 
Li, M., Li, S., Liao, H., Zeng, J., and Wang, Q. (2016). Spanwise correlation of aerodynamic forces on oscillating rectangular cylinder. Journal of Wind Engineering and Industrial Aerodynamics, 154:47-57.

Marra, A. M., Mannini, C., and Bartoli, G. (2011). Van der pol-type equation for modeling vortex-induced oscillations of bridge decks. Journal of Wind Engineering and Industrial Aerodynamics, 99:776-785.

Marra, A. M., Mannini, C., and Bartoli, G. (2015). Measurements and improved model of vortex-induced vibration for an elongated rectangular cylinder. Journal of Wind Engineering and Industrial Aerodynamics, 147:358-367.

Matsumoto, M., Shirato, H., Araki, K., Haramura, T., and Hashimoto, T. (2003). Spanwise coherence characteristics of surface pressure field on 2-d bluff bodies. Journal of Wind Engineering and Industrial Aerodynamics, 91:155-163.

Matsumoto, M., Yagi, T., Tamaki, H., and Tsubota, T. (2008). Vortex-induced vibration and its effect on torsional flutter instability in the case of $\mathrm{b} / \mathrm{d}=4$ rectangular cylinder. Journal of Wind Engineering and Industrial Aerodynamics, 96:971-983.

Mizota, T. and Okajima, A. (1981). Experimental studies of unsteady flows around rectangular prisms. In Proceedings of the Japan Society of Civil Engineers, volume 312, pages 49-57.

Nakaguchi, H., Hashimoto, K., and Muto, S. (1968). An experimental study on aerodynamic drag of rectangular cylinder. Journal of the Japan Society of Aeronautical Engineering, 16(168):1-5.

Nguyen, D. T., Hargreaves, D. M., and Owen, J. S. (2018). Vortex-induced vibration of a 5:1 rectangular cylinder: A comparison of wind tunnel sectional model tests and computational simulations. Journal of Wind Engineering and Industrial Aerodynamics, 175:1-16.

Ohya, Y., Nakamura, Y., Ozono, S., and Tsuruta, H. (1992). A numerical study of vortex shedding form flat plates with square leading and trailing edges. Journal of Fluids and Structures, 236:445-460.

Okajima, A. (1983). Flow around a rectangular cylinder with a section of various width/height ratios. Journal of Wind Engineering, Japan Association of Wind Engineering, 17:1-19.

Patruno, L. (2016). Numerical simulation of a 5:1 rectangular cylinder at non-null angles of attack. Journal of Wind Engineering and Industrial Aerodynamics, 151:146-157.

Ricci, M., Patruno, L., de Miranda, S., and Ubertini, F. (2017). Flow field around a 5:1 rectangular cylinder using les: Influence of inflow turbulence conditions, spanwise domain size and their interaction. Computers \& Fluids, 149:181-193.

Ricciardelli, F. (2010). Effects of the vibration regime on the spanwise correlation of the aerodynamic forces on a 5:1 rectangular cylinder. Journal of Wind Engineering and Industrial Aerodynamics, 98:215-225.

Sarkic, A. (2014). Validated numerical simulation of fluid structure interactions of bridge girders in turbulent wind fields. PhD thesis, Ruhr-Universität, Bochum, Germany.

Sarwar, M. W. and Ishihara, T. (2010). Numerical study on suppression of vortex-induced vibrations of box girder bridge section by aerodynamic countermeasures. Journal of Wind Engineering and Industrial Aerodynamics, 98:701-711.

Shimada, K. and Ishihara, T. (2012). Predictability of unsteady two-dimensional $k$ - $\epsilon$ model on the aerodynamic instabilities of some rectangular prisms. Journal of Fluids and Structures, 28:20-39.

Sohankar, A. (2008). Large eddy simulation of flow past rectangular-section cylinders: Side ratio effects. Journal of Wind Engineering and Industrial Aerodynamics, 96:640-655.

Vairo, G. (2003). A numerical model for wind loads simulation on long-span bridges. Simulation Modelling Practice and Theory, 11:315-351.

Washizu, K., Ohya, A., Otsuki, Y., and Fujii, K. (1978). Aeroelastic instability of rectangular cylinders in a heaving mode. Journal of Sound and Vibration, 59(2):195-210. 
Wilcox, D. C. (2012). Basic fluid mecanics. DCW Industries, La Cañada, fifth edition.

Wilkinson, R. H. (1981). Fluctuating pressures on an oscillating square prism. part ii. spanwise correlation and loading. Aeronautical Quarterly, 32(Part 2):111-125.

Xu, Y. L. (2013). Wind effects of cable-supported bridges. John Wiley \& Sons, Singapore, 1 edition.

Yoshizawa, A. (1986). Statistical theory for compressible shear flows, with the application of subgrid modelling. Physics of Fluids, 29(7):155-163.

$\mathrm{Yu}$, D. and Kareem, A. (1998). Parametric study of flow around rectangular prisms usign LES. Journal of Wind Engineering and Industrial Aerodynamics, 77-78:653-662. 